\title{
Rényi squashed entanglement, discord, and relative entropy differences
}

\author{
Kaushik P. Seshadreesan* Mario Berta ${ }^{\dagger} \quad$ Mark M. Wilde*‡
}

September 21, 2015

\begin{abstract}
The squashed entanglement quantifies the amount of entanglement in a bipartite quantum state, and it satisfies all of the axioms desired for an entanglement measure. The quantum discord is a measure of quantum correlations that are different from those due to entanglement. What these two measures have in common is that they are both based upon the conditional quantum mutual information. In [Berta et al., J. Math. Phys. 56, 022205 (2015)], we recently proposed Rényi generalizations of the conditional quantum mutual information of a tripartite state on $A B C$ (with $C$ being the conditioning system), which were shown to satisfy some properties that hold for the original quantity, such as non-negativity, duality, and monotonicity with respect to local operations on the system $B$ (with it being left open to show that the Rényi quantity is monotone with respect to local operations on system $A$ ). Here we define a Rényi squashed entanglement and a Rényi quantum discord based on a Rényi conditional quantum mutual information and investigate these quantities in detail. Taking as a conjecture that the Rényi conditional quantum mutual information is monotone with respect to local operations on both systems $A$ and $B$, we prove that the Rényi squashed entanglement and the Rényi quantum discord satisfy many of the properties of the respective original von Neumann entropy based quantities. In our prior work [Berta et al., Phys. Rev. A 91, 022333 (2015)], we also detailed a procedure to obtain Rényi generalizations of any quantum information measure that is equal to a linear combination of von Neumann entropies with coefficients chosen from the set $\{-1,0,1\}$. Here, we extend this procedure to include differences of relative entropies. Using the extended procedure and a conjectured monotonicity of the Rényi generalizations in the Rényi parameter, we discuss potential remainder terms for well known inequalities such as monotonicity of the relative entropy, joint convexity of the relative entropy, and the Holevo bound.
\end{abstract}

\section{Introduction}

Quantum information theory is intimately connected to the theory of quantum correlations. Information quantities such as the quantum entropy, conditional entropy, mutual information, and conditional mutual information underlie several measures of quantum correlations. For example, both the squashed entanglement [CW04 and the quantum discord OZ01 are based upon the

\footnotetext{
${ }^{*}$ Hearne Institute for Theoretical Physics and Department of Physics and Astronomy, Louisiana State University, Baton Rouge, Louisiana 70803, USA

${ }^{\dagger}$ Institute for Quantum Information and Matter, California Institute of Technology, Pasadena, California 91125, USA

${ }^{\ddagger}$ Center for Computation and Technology, Louisiana State University, Baton Rouge, Louisiana 70803, USA
} 
conditional quantum mutual information. Also, the operational meanings that the entropic quantities take in information-theoretic protocols aid in understanding the correlation measures defined based on them. The entropy finds operational meaning in the context of quantum data compression [Sch95], the conditional entropy in quantum state merging [HOW05, HOW07, the mutual information in erasure of total correlations [GPW05], and the conditional mutual information in quantum state redistribution [DY08, YD09].

The squashed entanglement of a bipartite state $\rho_{A B}$ is defined as [CW04]

$$
E^{\mathrm{sq}}(A ; B)_{\rho} \equiv \frac{1}{2} \inf _{\omega_{A B E}}\left\{I(A ; B \mid E)_{\omega}: \operatorname{Tr}_{E}\left\{\omega_{A B E}\right\}=\rho_{A B}\right\}
$$

where the infimum is with respect to all extensions $\omega_{A B E}$ of the state $\rho_{A B}$. The conditional mutual information $I(A ; B \mid E)_{\omega}$ in (1.1) is the linear combination

$$
I(A ; B \mid E)_{\omega} \equiv H(A E)_{\omega}+H(B E)_{\omega}-H(E)_{\omega}-H(A B E)_{\omega},
$$

with $H(F)_{\sigma} \equiv-\operatorname{Tr}\left\{\sigma_{F} \log \sigma_{F}\right\}$ being the von Neumann entropy of a state $\sigma_{F}$ on system $F$, where we unambiguously let $\omega_{E} \equiv \operatorname{Tr}_{A B}\left\{\omega_{A B E}\right\}$ denote the reduced density operator on system $E$, for example. The squashed entanglement satisfies all the desired properties of an entanglement measure. It is convex, monotone with respect to local operations and classical communication (LOCC), non-negative, superadditive in general, and additive on tensor-product states [CW04. It is also asymptotically continuous [AF04, and faithful (i.e., it takes the value zero if and only if the state $\rho_{A B}$ is separable) [CW04, BCY11. The squashed entanglement finds application as an upper bound on distillable entanglement and as a lower bound on entanglement cost [CW04. The squashed entanglement of a quantum channel is defined as the maximum squashed entanglement that can be registered by a sender and a receiver at the input and output of a channel [TGW14b, TGW14a]. This quantity is an upper bound on the quantum communication capacity of any channel assisted by unlimited forward and backward classical communication as well as the private capacity of a quantum channel assisted by unlimited forward and backward public classical communication.

The quantum discord of a bipartite state $\rho_{A B}$ is defined as the gap between the quantum mutual information $I(A ; B)_{\rho} \equiv H(A)_{\rho}+H(B)_{\rho}-H(A B)_{\rho}$ and the mutual information after one of the systems $A$ or $B$ has been measured, where the latter is maximized over all measurements [Zur00, OZ01]. That is, if the measurement occurs on system $A$, then the quantum discord is defined as

$$
D(\bar{A} ; B)_{\rho} \equiv I(A ; B)_{\rho}-\max _{\left\{\Lambda^{x}\right\}} I(X ; B)_{\tau},
$$

with the overbar denoting the system being measured,

$$
\tau_{X B} \equiv \sum_{x}|x\rangle\left\langle\left. x\right|_{X} \otimes \operatorname{Tr}_{A}\left\{\left(\Lambda_{A}^{x} \otimes I_{B}\right) \rho_{A B}\right\},\right.
$$

$\Lambda^{x} \geq 0 \forall x, \sum_{x} \Lambda^{x}=I$, and $\{|x\rangle\}$ is an orthonormal basis. (It is well known to be sufficient to optimize the quantum discord with respect to rank-one POVMs $\mathrm{MBC}^{+} 12$.) We think that it is an important conceptual realization that the quantum discord can be re-expressed as the following conditional quantum mutual information [Pia12]:

$$
D(\bar{A} ; B)_{\rho}=\inf _{\left\{\Lambda^{x}\right\}} I(E ; B \mid X)_{\tau}
$$


where the optimization is with respect to all possible POVMs acting on system $A$, with $X$ being the classical output and $E$ being an environment for the measurement map, so that

$$
\begin{aligned}
\tau_{X E B} & \equiv U_{A \rightarrow X E} \rho_{A B} U_{A \rightarrow X E}^{\dagger} \\
U_{A \rightarrow X E}|\psi\rangle_{A} & \equiv \sum_{x}|x\rangle_{X} \otimes\left(\sqrt{\Lambda^{x}}|\psi\rangle_{A} \otimes|x\rangle\right)_{E} .
\end{aligned}
$$

The quantum discord characterizes quantum correlations that are different from those due to entanglement. It is non-negative, invariant with respect to local unitary operations, and equal to zero if and only if the state is classical on the system being measured $\mathrm{MBC}^{+} 12$.

In quantum information theory, there has been interest recently in generalizing information measures in terms of the Rényi entropies. Such Rényi generalizations of information measures enable an improved understanding of the original von Neumann entropy-based measures and also find applications in scenarios beyond the traditional independent and identically (i.i.d.) distributed resource setting [Pet86, Ren05, Dat09, MH11, Tom12, MLDS ${ }^{+13}$, WWY14]. Since quantum information quantities underlie some quantum correlation measures, it is natural to think of Rényi generalizations of those correlation measures as well.

In our prior work [BSW15b], we had proposed a procedure to obtain Rényi generalizations of quantum information measures that are equal to linear combinations of von Neumann entropies with coefficients chosen from the set $\{-1,0,1\}$. In particular, Rényi generalizations of the conditional quantum mutual information of (1.2) were proposed [BSW15a, Sections 5 and 6]. These generalizations retain most of the desired properties of the von Neumann entropy-based conditional quantum mutual information; e.g., they are non-negative, monotone with respect to local quantum operations on one of the two systems $A$ or $B$, and obey a duality property for four-party pure states $|\psi\rangle_{A B C D}$ [BSW15a, Section 7]. There it was left as an open question to determine whether monotonicity also holds for local operations on the other system, with numerical evidence supporting a positive answer.

In this work, based on the Rényi conditional quantum mutual informations, we define and explore the properties of Rényi generalizations of the squashed entanglement and the quantum discord.112 Additionally, we extend the scope of the procedure for Rényi generalization of quantum information measures prescribed in our former work to include quantum information measures which are expressed as differences of relative entropies. The major potential application of this latter contribution is in finding "remainder terms" for monotonicity of relative entropy, joint convexity of relative entropy, and the Holevo bound.

\section{Overview of results}

The main purpose of the present paper is to justify the Rényi squashed entanglement and quantum discord quantities introduced here as useful measures of quantum correlation. Complementary to this aim, we take the results here as further justification that [BSW15a] has pinned down a useful Rényi generalization of the conditional mutual information. Furthermore, our work suggests

\footnotetext{
${ }^{1}$ Note that what we define in this paper as 'the' Rényi squashed entanglement and 'the' Rényi quantum discord are only one of many possible definitions for the quantities based on the many different Rényi conditional mutual informations defined in BSW15a].

${ }^{2} \mathrm{~A}$ different Rényi generalization of the quantum discord based on a difference of Rényi mutual informations was proposed and studied in $\left[\mathrm{MBP}^{+} 15\right]$.
} 
that the axiomatic approach to entanglement theory and discord does not uniquely single out the von Neumann entropy-based squashed entanglement or discord (however, further effort is needed to justify this claim in full). Finally, a potential application for future work is to use the Rényi quantities in order to refine information-theoretic results concerning the original von Neumann entropy-based quantities.

At the outset, it is important to note that this paper takes as a conjecture that the Rényi conditional quantum mutual information is monotone with respect to local quantum operations on both systems $A$ and $B$ (see Conjecture 1). As already stated in Section 1, the aforementioned monotonicity was proven in BSW15a for local quantum operations on one of the two systems $A$ or $B$, and it was left as an open question to determine if the property holds for local quantum operations on the other system (with numerical evidence supporting a positive answer). Here, we assume the latter to hold. Some of the results presented in this work are based upon this conjecture.

Our contributions are as follows:

- We show that the Rényi squashed entanglement defined here retains many of the desired properties of the original von Neumann entropy based quantity, such as monotonicity with respect to LOCC (up to the conjecture on the monotonicity of the Rényi conditional quantum mutual information with respect to local operations), convexity, and sub-additivity on tensorproduct states. These properties hold only for particular regimes of the Rényi parameter $\alpha$. The first two properties together qualify the Rényi squashed entanglement as an entanglement monotone, again for certain regimes of $\alpha$ and up to the conjecture.

- The squashed entanglement is known to be bounded from above by the entanglement of formation [CW04. Here we define a Rényi entanglement of formation based on the Rényi conditional entropy, and show that the $\alpha$-Rényi squashed entanglement is also upper bounded by a $\beta$-Rényi entanglement of formation, where $\beta=(2-\alpha) / \alpha$.

- We show that the Rényi discord is non-negative, invariant with respect to local unitaries, and equal to zero if the state is a classical-quantum state. Furthermore, we prove that it suffices to optimize the Rényi discord functional with respect to rank-one measurements. We then discuss an operational characterization of bipartite quantum states with small von Neumann entropy-based quantum discord, which would follow from [BSW15a, Conjecture 34] (see just after (3.20) for the formal statement of this conjecture).

- Finally, we extend the procedure to obtain Rényi generalizations of quantum information measures given in our prior work [BSW15b] by showing how to obtain Rényi generalizations of differences of relative entropies. The main purpose of this last contribution is to study potential remainder terms for monotonicity of the relative entropy, joint convexity of the relative entropy, and the Holevo bound.

\section{Preliminaries}

Norms, states, extensions, channels, and measurements. Let $\mathcal{B}(\mathcal{H})$ denote the algebra of bounded linear operators acting on a Hilbert space $\mathcal{H}$. We restrict ourselves to finite-dimensional Hilbert spaces throughout this paper. For $\alpha \geq 1$, we define the $\alpha$-norm of an operator $X \in \mathcal{B}(\mathcal{H})$ as

$$
\|X\|_{\alpha} \equiv\left[\operatorname{Tr}\left\{|X|^{\alpha}\right\}\right]^{1 / \alpha}
$$


where $|X| \equiv \sqrt{X^{\dagger} X}$ and we use the same notation even for the case $\alpha \in(0,1)$, when it is not a norm. Let $\mathcal{B}(\mathcal{H})_{+}$denote the subset of positive semi-definite operators, and let $\mathcal{B}(\mathcal{H})_{++}$denote the subset of positive definite operators. We also write $X \geq 0$ if $X \in \mathcal{B}(\mathcal{H})_{+}$and $Y>0$ if $Y \in \mathcal{B}(\mathcal{H})_{++}$. An operator $\rho$ is in the set $\mathcal{S}(\mathcal{H})$ of density operators (or states) if $\rho \in \mathcal{B}(\mathcal{H})_{+}$and $\operatorname{Tr}\{\rho\}=1$. An operator $\rho$ is in the set $\mathcal{S}(\mathcal{H})_{++}$if it is a density operator and positive definite. The tensor product of two Hilbert spaces $\mathcal{H}_{A}$ and $\mathcal{H}_{B}$ is denoted by $\mathcal{H}_{A} \otimes \mathcal{H}_{B}$ or $\mathcal{H}_{A B}$. Given a multipartite density operator $\rho_{A B} \in \mathcal{S}\left(\mathcal{H}_{A} \otimes \mathcal{H}_{B}\right)$, we unambiguously write $\rho_{A}=\operatorname{Tr}_{B}\left\{\rho_{A B}\right\}$ for the reduced density operator on system $A$. We use $\rho_{A B}, \sigma_{A B}, \tau_{A B}, \omega_{A B}$, etc. to denote general density operators in $\mathcal{S}\left(\mathcal{H}_{A} \otimes \mathcal{H}_{B}\right)$, while $\psi_{A B}, \varphi_{A B}, \phi_{A B}$, etc. denote rank-one density operators (pure states) in $\mathcal{S}\left(\mathcal{H}_{A} \otimes \mathcal{H}_{B}\right)$ (with it implicit, clear from the context, and the above convention implying that $\psi_{A}, \varphi_{A}, \phi_{A}$ are mixed if $\psi_{A B}, \varphi_{A B}, \phi_{A B}$ are pure and entangled).

We also say that pure-state vectors $|\psi\rangle$ in $\mathcal{H}$ are states. Any bipartite pure state $|\psi\rangle_{A B}$ in $\mathcal{H}_{A B}$ is written in Schmidt form as

$$
|\psi\rangle_{A B} \equiv \sum_{i=0}^{d-1} \sqrt{\lambda_{i}}|i\rangle_{A}|i\rangle_{B},
$$

where $\left\{|i\rangle_{A}\right\}$ and $\left\{|i\rangle_{B}\right\}$ form orthonormal bases in $\mathcal{H}_{A}$ and $\mathcal{H}_{B}$, respectively, $d$ is the Schmidt rank of the state, and $\sum_{i=0}^{d-1} \lambda_{i}=1$. By a maximally entangled state, we mean a bipartite pure state of the form

$$
|\Phi\rangle_{A B} \equiv \frac{1}{\sqrt{d}} \sum_{i=0}^{d-1}|i\rangle_{A}|i\rangle_{B} .
$$

The trace distance between two quantum states $\rho, \sigma \in \mathcal{S}(\mathcal{H})$ is equal to $\|\rho-\sigma\|_{1}$. It has a direct operational interpretation in terms of the distinguishability of these states. That is, if $\rho$ or $\sigma$ are prepared with equal probability and the task is to distinguish them via some quantum measurement, then the optimal success probability in doing so is equal to $\left(1+\|\rho-\sigma\|_{1} / 2\right) / 2$.

Throughout this paper, we take the usual convention that $f(A)=\sum_{i: a_{i}>0} f\left(a_{i}\right)|i\rangle\langle i|$ when given a function $f$ and a positive semi-definite operator $A$ with spectral decomposition $A=$ $\sum_{i} a_{i}|i\rangle\langle i|$. So this means that $A^{-1}$ is interpreted as a generalized inverse, so that $A^{-1}=$ $\sum_{i: a_{i}>0} a_{i}^{-1}|i\rangle\left\langle i\left|, \log (A)=\sum_{i: a_{i}>0} \log \left(a_{i}\right)\right| i\right\rangle\left\langle i\left|, \exp (A)=\sum_{i: a_{i}>0} \exp \left(a_{i}\right)\right| i\right\rangle\langle i|$, etc. The above convention for $f(A)$ leads to the convention that $A^{0}$ denotes the projection onto the support of $A$, i.e., $A^{0}=\sum_{i: a_{i}>0}|i\rangle\langle i|$. We employ the shorthand $\operatorname{supp}(A)$ to refer to the support of an operator $A$.

A linear map $\mathcal{N}_{A \rightarrow B}: \mathcal{B}\left(\mathcal{H}_{A}\right) \rightarrow \mathcal{B}\left(\mathcal{H}_{B}\right)$ is positive if $\mathcal{N}_{A \rightarrow B}\left(\sigma_{A}\right) \in \mathcal{B}\left(\mathcal{H}_{B}\right)_{+}$whenever $\sigma_{A} \in$ $\mathcal{B}\left(\mathcal{H}_{A}\right)_{+}$. Let id $A_{A}$ denote the identity map acting on a system $A$. A linear map $\mathcal{N}_{A \rightarrow B}$ is completely positive if the map id $R \otimes \mathcal{N}_{A \rightarrow B}$ is positive for a reference system $R$ of arbitrary size. A linear map $\mathcal{N}_{A \rightarrow B}$ is strictly positive if it takes positive definite operators on the input to positive definite operators on the output, and it is a strict completely positive map if $\operatorname{id}_{R} \otimes \mathcal{N}_{A \rightarrow B}$ is strictly positive for a reference system $R$ of arbitrary size. A linear map $\mathcal{N}_{A \rightarrow B}$ is trace-preserving if $\operatorname{Tr}\left\{\mathcal{N}_{A \rightarrow B}\left(\tau_{A}\right)\right\}=\operatorname{Tr}\left\{\tau_{A}\right\}$ for all input operators $\tau_{A} \in \mathcal{B}\left(\mathcal{H}_{A}\right)$. If a linear map is completely positive and trace-preserving (CPTP), we say that it is a quantum channel or quantum operation. A positive operator-valued measure (POVM) is a set $\left\{\Lambda^{m}\right\}$ of positive semi-definite operators such that $\sum_{m} \Lambda^{m}=I$.

An extension of a state $\rho_{A} \in \mathcal{S}\left(\mathcal{H}_{A}\right)$ is some state $\Omega_{R A} \in \mathcal{S}\left(\mathcal{H}_{R} \otimes \mathcal{H}_{A}\right)$ such that $\operatorname{Tr}_{R}\left\{\Omega_{R A}\right\}=$ $\rho_{A}$. An isometric extension $U_{A \rightarrow B E}^{\mathcal{N}}$ of a channel $\mathcal{N}_{A \rightarrow B}$ acting on a state $\rho_{A} \in \mathcal{S}\left(\mathcal{H}_{A}\right)$ is a linear map that satisfies the following:

$$
\operatorname{Tr}_{E}\left\{U_{A \rightarrow B E}^{\mathcal{N}} \rho_{A}\left(U_{A \rightarrow B E}^{\mathcal{N}}\right)^{\dagger}\right\}=\mathcal{N}_{A \rightarrow B}\left(\rho_{A}\right), \quad U_{\mathcal{N}}^{\dagger} U_{\mathcal{N}}=I_{A}, \quad U_{\mathcal{N}} U_{\mathcal{N}}^{\dagger}=\Pi_{B E}
$$


where $\Pi_{B E}$ is a projection onto a subspace of the Hilbert space $\mathcal{H}_{B} \otimes \mathcal{H}_{E}$.

Rényi entropies and information measures. The $\alpha$-Rényi entropy and the $\alpha$-Rényi divergence, are defined respectively for a parameter $\alpha \in(0,1) \cup(1, \infty)$ and probability distributions $p$ and $q$ as

$$
\begin{aligned}
H_{\alpha}(p) & \equiv \frac{1}{1-\alpha} \log \sum_{x}[p(x)]^{\alpha}, \\
D_{\alpha}(p \| q) & \equiv \frac{1}{\alpha-1} \log \sum_{x}[p(x)]^{\alpha}[q(x)]^{1-\alpha},
\end{aligned}
$$

where log denotes the natural logarithm here and throughout the paper. The Shannon entropy and relative entropy are recovered in the limit as $\alpha \rightarrow 1$ :

$$
\begin{gathered}
\lim _{\alpha \rightarrow 1} H_{\alpha}(p)=H(p) \equiv-\sum_{x} p(x) \log p(x), \\
\lim _{\alpha \rightarrow 1} D_{\alpha}(p \| q)=D(p \| q) \equiv \sum_{x} p(x) \log \frac{p(x)}{q(x)} .
\end{gathered}
$$

The quantum Rényi entropy of a state $\rho$ is defined for $\alpha \in(0,1) \cup(1, \infty)$ as

$$
H_{\alpha}(\rho) \equiv \frac{1}{1-\alpha} \log \operatorname{Tr}\left\{\rho^{\alpha}\right\}=\frac{\alpha}{1-\alpha} \log \|\rho\|_{\alpha},
$$

and reduces to the von Neumann entropy in the limit as $\alpha \rightarrow 1$ :

$$
\lim _{\alpha \rightarrow 1} H_{\alpha}(\rho)=H(\rho) .
$$

There are at least two ways to generalize the quantum relative entropy. The Rényi relative entropy of order $\alpha \in[0,1) \cup(1, \infty)$ is defined as Pet86]

$$
D_{\alpha}(\rho \| \sigma) \equiv\left\{\begin{array}{c}
\frac{1}{\alpha-1} \log \operatorname{Tr}\left\{\begin{array}{c}
\left.\operatorname{Tr}\{\rho\}]^{-1} \rho^{\alpha} \sigma^{1-\alpha}\right\} \\
+\infty
\end{array} \quad \text { if } \operatorname{supp}(\rho) \subseteq \operatorname{supp}(\sigma) \text { or }(\alpha \in[0,1) \text { and } \rho \not L \sigma)\right. \\
\text { otherwise }
\end{array},\right.
$$

with the support conditions established in TCR09]. The Rényi relative entropy obeys monotonicity with respect to quantum operations for $\alpha \in[0,1) \cup(1,2]$ [Pet86], in the sense that $D_{\alpha}(\rho \| \sigma) \geq$ $D_{\alpha}(\mathcal{N}(\rho) \| \mathcal{N}(\sigma))$ for a quantum operation $\mathcal{N}$.

The sandwiched Rényi relative entropy [MLDS ${ }^{+} 13$, WWY14] is another variant of the Rényi relative entropy which has found a number of applications recently in the context of strong converse theorems [WWY14, MO15, GW15, CMW14, TWW14]. It is defined for $\alpha \in(0,1) \cup(1, \infty)$ as follows:

$$
\widetilde{D}_{\alpha}(\rho \| \sigma) \equiv\left\{\begin{array}{cc}
\frac{1}{\alpha-1} \log \left[[\operatorname{Tr}\{\rho\}]^{-1} \operatorname{Tr}\left\{\left(\sigma^{(1-\alpha) / 2 \alpha} \rho \sigma^{(1-\alpha) / 2 \alpha}\right)^{\alpha}\right\}\right] & \text { if } \operatorname{supp}(\rho) \subseteq \operatorname{supp}(\sigma) \text { or } \\
+\infty & (\alpha \in(0,1) \text { and } \rho \not \perp \sigma) \\
\text { otherwise }
\end{array}\right.
$$

The Rényi conditional entropy for a bipartite state $\rho_{A B}$ is defined for $\alpha \in(0,1) \cup(1, \infty)$ as

$$
\begin{aligned}
H_{\alpha}(A \mid B) & \equiv-\min _{\sigma_{B}} D_{\alpha}\left(\rho_{A B} \| I_{A} \otimes \sigma_{B}\right) \\
& =\frac{\alpha}{1-\alpha} \log \operatorname{Tr}\left\{\left(\operatorname{Tr}_{A}\left\{\rho_{A B}^{\alpha}\right\}\right)^{1 / \alpha}\right\},
\end{aligned}
$$


where $D_{\alpha}$ is the Rényi relative entropy defined in (3.11) and the second equality follows from a Sibson identity [SW13]. The Rényi quantum mutual information of a bipartite state $\rho_{A B}$ is defined for $\alpha \in(0,1) \cup(1, \infty)$ as

$$
\begin{aligned}
I_{\alpha}(A ; B)_{\rho} & \equiv \min _{\sigma_{B}} D_{\alpha}\left(\rho_{A B} \| \rho_{A} \otimes \sigma_{B}\right) \\
& =\frac{\alpha}{\alpha-1} \log \operatorname{Tr}\left\{\left(\operatorname{Tr}_{A}\left\{\rho_{A}^{1-\alpha} \rho_{A B}^{\alpha}\right\}\right)^{\frac{1}{\alpha}}\right\},
\end{aligned}
$$

where $D_{\alpha}$ is the Rényi relative entropy defined in (3.11) and the second equality follows from a Sibson identity [GW15, Corollary 8]. Throughout this paper, we take our definition of Rényi conditional quantum mutual information for $\alpha \in(0,1) \cup(1, \infty)$ to be as follows [BSW15a, Definition 7]:

$$
\begin{aligned}
I_{\alpha}(A ; B \mid E)_{\rho} & \equiv \inf _{\sigma_{B E}} D_{\alpha}\left(\rho_{A B E} \|\left(\rho_{A E}^{(1-\alpha) / 2} \rho_{E}^{(\alpha-1) / 2} \sigma_{B E}^{1-\alpha} \rho_{E}^{(\alpha-1) / 2} \rho_{A E}^{(1-\alpha) / 2}\right)^{\frac{1}{1-\alpha}}\right) \\
& =\frac{\alpha}{\alpha-1} \log \operatorname{Tr}\left\{\left(\rho_{E}^{(\alpha-1) / 2} \operatorname{Tr}_{A}\left\{\rho_{A E}^{(1-\alpha) / 2} \rho_{A B E}^{\alpha} \rho_{A E}^{(1-\alpha) / 2}\right\} \rho_{E}^{(\alpha-1) / 2}\right)^{1 / \alpha}\right\},
\end{aligned}
$$

where $D_{\alpha}$ is the Rényi relative entropy defined in (3.11) and the second equality follows from a Sibson identity [BSW15a, Proposition 8]. The Rényi conditional quantum mutual information is non-negative and obeys monotonicity with respect to local CPTP maps on system $B$, i.e., for $\alpha \in(0,1) \cup(1,2]$,

$$
I_{\alpha}(A ; B \mid E)_{\rho} \geq I_{\alpha}\left(A ; B^{\prime} \mid E\right)_{\omega},
$$

where $\omega_{A B^{\prime} E} \equiv \mathcal{N}_{B \rightarrow B^{\prime}}\left(\rho_{A B E}\right)$ and $\mathcal{N}_{B \rightarrow B^{\prime}}$ is any CPTP map acting on system $B$ alone. For a four-party pure state $\phi_{A B C D}$, the Rényi conditional quantum mutual information satisfies a duality property [BSW15a, Theorem 32] given by

$$
I_{\alpha}(A ; B \mid C)_{\phi}=I_{\alpha}(B ; A \mid D)_{\phi} .
$$

It is conjectured [BSW15a, Conjecture 34] that the Rényi conditional mutual information $I_{\alpha}(A ; B \mid E)$ is monotone non-decreasing with respect to the Rényi parameter $\alpha$.

Some results in this paper rely on the following conjecture:

Conjecture 1 The Rényi conditional quantum mutual information obeys monotonicity with respect to CPTP maps on system $A$, i.e., for $\rho_{A B E} \in \mathcal{S}\left(\mathcal{H}_{A B E}\right)$ and $\alpha \in(0,1) \cup(1,2]$,

$$
I_{\alpha}(A ; B \mid E)_{\rho} \geq I_{\alpha}\left(A^{\prime} ; B \mid E\right)_{\tau},
$$

where $\tau_{A^{\prime} B E} \equiv \mathcal{M}_{A \rightarrow A^{\prime}}\left(\rho_{A B E}\right)$ and $\mathcal{M}_{A \rightarrow A^{\prime}}$ is a CPTP map acting on system $A$ alone.

\section{Rényi Squashed Entanglement}

In this section, we explore a Rényi squashed entanglement, assuming Conjecture 1. We show various properties of the quantity which justify it is a valid entanglement measure (again assuming Conjecture 1). We also establish a relation between the Rényi squashed entanglement and the Rényi entanglement of formation. We end the section with Table 1, which summarizes the properties of the Rényi squashed entanglement and draws a comparison with those of the von Neumann entropy based squashed entanglement. 
Definition 2 The Rényi squashed entanglement of $\rho_{A B} \in \mathcal{S}\left(\mathcal{H}_{A B}\right)$ is defined for $\alpha \in(0,1) \cup(1, \infty)$ as

$$
E_{\alpha}^{\mathrm{sq}}(A ; B)_{\rho} \equiv \frac{1}{2} \inf _{\omega_{A B E}}\left\{I_{\alpha}(A ; B \mid E)_{\omega}: \rho_{A B}=\operatorname{Tr}_{E}\left\{\omega_{A B E}\right\}\right\}
$$

where the infimum is with respect to all extensions $\omega_{A B E}$ of the state $\rho_{A B}$ and $I_{\alpha}(A ; B \mid E)_{\omega}$ is the Rényi conditional quantum mutual information from (3.18).

\subsection{Entanglement monotone}

Assuming Conjecture 1, we now show that the Rényi squashed entanglement of Definition 2 is an entanglement monotone for $\alpha \in(0,1)$ (see [PV07, HHHH09] for a review of the axiomatic approach to entanglement measures). We say that a quantity is an entanglement monotone if it is an LOCC monotone and convex [Vid00. We prove that the Rényi squashed entanglement is an LOCC monotone for $\alpha \in(0,1) \cup(1,2]$ (assuming Conjecture 11) and convex for $\alpha \in(0,1)$. Before beginning, we recall that local operations and classical communication consists of compositions of the following operations:

1. Alice performs a quantum instrument, which has both a quantum and classical output. She forwards the classical output to Bob, who then performs a quantum operation conditioned on the classical data received.

2. The situation is reversed, with Bob performing the initial instrument, who forwards the classical data to Alice, who then performs a quantum operation conditioned on the classical data.

Thus, in order to establish a measure as an LOCC monotone, it suffices to show that it is nonincreasing with respect to local operations and invariant with respect to the sharing of classical data.

Consequence 3 Assuming Conjecture 1, the Rényi squashed entanglement $E_{\alpha}^{\mathrm{sq}}(A ; B)_{\rho}$ is monotone with respect to local operations for $\alpha \in(0,1) \cup(1,2]$ :

$$
E_{\alpha}^{\mathrm{sq}}(A ; B)_{\rho} \geq E_{\alpha}^{\mathrm{sq}}\left(A^{\prime} ; B^{\prime}\right)_{\sigma}
$$

where $\rho_{A B} \in \mathcal{S}\left(\mathcal{H}_{A B}\right), \sigma_{A^{\prime} B^{\prime}} \equiv\left(\mathcal{N}_{A \rightarrow A^{\prime}} \otimes \mathcal{M}_{B \rightarrow B^{\prime}}\right)\left(\rho_{A B}\right)$, and $\mathcal{N}_{A \rightarrow A^{\prime}}, \mathcal{M}_{B \rightarrow B^{\prime}}$ are CPTP maps.

Proof. This follows directly from monotonicity with respect to local operations of the Rényi conditional mutual information $I_{\alpha}(A ; B \mid E)_{\rho}$.

Consequence 4 Assuming Conjecture 1, the Rényi squashed entanglement $E_{\alpha}^{\mathrm{sq}}(A ; B)_{\rho}$ is invariant with respect to classical communication for $\alpha \in(0,1) \cup(1,2]$, in the sense that

$$
E_{\alpha}^{\mathrm{sq}}\left(A X_{A} ; B\right)_{\rho}=E_{\alpha}^{\mathrm{sq}}\left(A X_{A} ; B X_{B}\right)_{\rho}=E_{\alpha}^{\mathrm{sq}}\left(A ; B X_{B}\right)_{\rho},
$$

where

$$
\rho_{X_{A} X_{B} A B} \equiv \sum_{x} p_{X}(x)|x\rangle\left\langle\left. x\right|_{X_{A}} \otimes \mid x\right\rangle\left\langle\left. x\right|_{X_{B}} \otimes \rho_{A B}^{x}\right.
$$


Proof. Let $\rho_{X_{A} A B E}$ be any extension of $\rho_{X_{A} A B}$, taking the form

$$
\sum_{x} p_{X}(x)|x\rangle\left\langle\left. x\right|_{X_{A}} \otimes \rho_{A B E}^{x},\right.
$$

where $\rho_{A B E}^{x}$ is an extension of $\rho_{A B}^{x}$. Let $\left|\varphi^{\rho}\right\rangle_{X_{A} X_{R} A B E R}$ be a purification of $\rho_{X_{A} A B E}$, taken as

$$
\left|\varphi^{\rho}\right\rangle_{X_{A} X_{R} A B E R} \equiv \sum_{x} \sqrt{p_{X}(x)}|x\rangle_{X_{A}}|x\rangle_{X_{R}}\left|\varphi^{\rho_{x}}\right\rangle_{A B E R}
$$

where $\left|\varphi^{\rho_{x}}\right\rangle_{A B E R}$ purifies $\rho_{A B E}^{x}$. Let $\bar{\varphi}_{X_{A} X_{B} X_{R} A B E R}$ be defined as

$$
\bar{\varphi}_{X_{A} X_{B} X_{R} A B E R}^{\rho} \equiv \sum_{x} p_{X}(x)|x\rangle\left\langle\left. x\right|_{X_{A}} \otimes \mid x\right\rangle\left\langle\left. x\right|_{X_{B}} \otimes \mid x\right\rangle\left\langle\left. x\right|_{X_{R}} \otimes \varphi_{A B E R}^{\rho_{x}} .\right.
$$

Let $\theta_{X_{A} X_{B} X_{R} A B E R F}$ be a purification of $\bar{\varphi}_{X_{A} X_{B} X_{R} A B E R}^{\rho}$. We then have that

$$
\begin{aligned}
I_{\alpha}\left(A X_{A} ; B \mid E\right)_{\rho} & =I_{\alpha}\left(B ; A X_{A} \mid X_{R} R\right)_{\varphi^{\rho}} \\
& \geq I_{\alpha}\left(B ; A X_{A} \mid X_{R} R\right)_{\bar{\varphi}^{\rho}} \\
& =\frac{\alpha}{\alpha-1} \log \sum_{x} p_{X}(x) \exp \left\{\left(\frac{\alpha-1}{\alpha}\right) I_{\alpha}\left(B ; A X_{A} \mid R\right)_{|x\rangle\langle x| \otimes \varphi^{\rho x}}\right\} \\
& =\frac{\alpha}{\alpha-1} \log \sum_{x} p_{X}(x) \exp \left\{\left(\frac{\alpha-1}{\alpha}\right) I_{\alpha}\left(B X_{B} ; A X_{A} \mid R\right)_{|x\rangle\langle x|\otimes| x\rangle\langle x| \otimes \varphi^{\rho_{x}}}\right\} \\
& =I_{\alpha}\left(B X_{B} ; A X_{A} \mid X_{R} R\right)_{\bar{\varphi}^{\rho}} \\
& =I_{\alpha}\left(A X_{A} ; B X_{B} \mid E F\right)_{\theta} \\
& \geq 2 E_{\alpha}^{\mathrm{sq}}\left(A X_{A} ; B X_{B}\right)_{\rho} .
\end{aligned}
$$

The first equality follows from duality of the Rényi conditional quantum mutual information given in (3.20). The first inequality is a result of monotonicity with respect to a local dephasing operation $\sum_{x}|x\rangle\left\langle\left. x\right|_{X_{A}}(\cdot) \mid x\right\rangle\left\langle\left. x\right|_{X_{A}}\right.$. The second equality follows from Lemma 31 given in Appendix A.2. The third equality is an application of Lemma 30 given in Appendix A.1. The fourth equality is from another application of Lemma 31. The fifth equality is from another application of duality given in (3.20). The final inequality is a result of Definition 2, Since the inequality holds for any extension of $\rho_{X_{A} A B}$, it follows that

$$
E_{\alpha}^{\mathrm{sq}}\left(A X_{A} ; B\right)_{\rho} \geq E_{\alpha}^{\mathrm{sq}}\left(A X_{A} ; B X_{B}\right)_{\rho} .
$$

By a similar line of reasoning (which relies on Conjecture 1), it follows that

$$
E_{\alpha}^{\mathrm{sq}}\left(A ; B X_{B}\right)_{\rho} \geq E_{\alpha}^{\mathrm{sq}}\left(A X_{A} ; B X_{B}\right)_{\rho} .
$$

Finally, from monotonicity of $E_{\alpha}^{\mathrm{sq}}$ with respect to local operations (which relies on Conjecture 1), we can conclude that

$$
\begin{aligned}
& E_{\alpha}^{\mathrm{sq}}\left(A X_{A} ; B X_{B}\right)_{\rho} \geq E_{\alpha}^{\mathrm{sq}}\left(A X_{A} ; B\right)_{\rho}, \\
& E_{\alpha}^{\mathrm{sq}}\left(A X_{A} ; B X_{B}\right)_{\rho} \geq E_{\alpha}^{\mathrm{sq}}\left(A ; B X_{B}\right)_{\rho} .
\end{aligned}
$$

So (4.15)-(4.18) give the statement of the lemma. 
Consequence 5 Assuming Conjecture 1 , the Rényi squashed entanglement $E_{\alpha}^{\mathrm{sq}}(A ; B)_{\rho}$ is an $L O C C$ monotone for $\alpha \in(0,1) \cup(1,2]$.

Proof. This consequence follows from Consequences 3 and 4 .

Proposition 6 The Rényi squashed entanglement $E_{\alpha}^{\mathrm{sq}}(A ; B)_{\rho}$ is convex for $\alpha \in(0,1)$, in the sense that

$$
\sum_{x} p_{X}(x) E_{\alpha}^{\mathrm{sq}}(A ; B)_{\rho^{x}} \geq E_{\alpha}^{\mathrm{sq}}(A ; B)_{\bar{\rho}}
$$

where

$$
\bar{\rho}_{A B} \equiv \sum_{x} p_{X}(x) \rho_{A B}^{x}
$$

Proof. Let $\rho_{A B E}^{x}$ be any extension of $\rho_{A B}^{x}$. Let

$$
\rho_{X A B E} \equiv \sum_{x} p_{X}(x)|x\rangle\left\langle\left. x\right|_{X} \otimes \rho_{A B E}^{x}\right.
$$

Observe that $\rho_{X A B E}$ is an extension of $\bar{\rho}_{A B}$, where the extension systems are $X$ and $E$. So then

$$
\begin{aligned}
2 E_{\alpha}^{\mathrm{sq}}(A ; B)_{\bar{\rho}} & \leq I_{\alpha}(A ; B \mid E X) \\
& =\frac{\alpha}{\alpha-1} \log \sum_{x} p_{X}(x) \exp \left\{\left(\frac{\alpha-1}{\alpha}\right) I_{\alpha}(A ; B \mid E)_{\rho^{x}}\right\} \\
& \leq \sum_{x} p_{X}(x)\left[\frac{\alpha}{\alpha-1} \log \exp \left\{\left(\frac{\alpha-1}{\alpha}\right) I_{\alpha}(A ; B \mid E)_{\rho^{x}}\right\}\right] \\
& =\sum_{x} p_{X}(x) I_{\alpha}(A ; B \mid E)_{\rho^{x}} .
\end{aligned}
$$

The first inequality follows from the definition of $E_{\alpha}^{\mathrm{sq}}(A ; B)_{\bar{\rho}}$, and the second inequality follows from convexity of $-\log$. Since the inequality holds for any extension of each $\rho_{A B E}^{x}$, we can conclude the statement of the proposition.

Consequence 7 Assuming Conjecture 1, the Rényi squashed entanglement $E_{\alpha}^{\mathrm{sq}}(A ; B)_{\rho}$ is an entanglement monotone for $\alpha \in(0,1)$.

Proof. This consequence follows from Consequence 5 and Proposition 6.

\subsection{Other properties}

Having shown that the Rényi squashed entanglement is an entanglement monotone (up to Conjecture 1), we now prove more properties of the quantity that bolster it as a valid entanglement measure.

Proposition 8 For separable states $\rho_{A B} \in \mathcal{S}\left(\mathcal{H}_{A B}\right)$ the Rényi squashed entanglement $E_{\alpha}^{\mathrm{sq}}(A ; B)_{\rho}$ vanishes for $\alpha \in(0,1) \cup(1, \infty)$. 
Proof. We can take a convex decomposition of any separable state as follows:

$$
\rho_{A B}=\sum_{x} p_{X}(x)\left|\psi_{x}\right\rangle\left\langle\left.\psi_{x}\right|_{A} \otimes \mid \phi_{x}\right\rangle\left\langle\left.\phi_{x}\right|_{B}\right.
$$

Then an extension is

$$
\rho_{A B X}=\sum_{x} p_{X}(x)\left|\psi_{x}\right\rangle\left\langle\left.\psi_{x}\right|_{A} \otimes \mid \phi_{x}\right\rangle\left\langle\left.\phi_{x}\right|_{B} \otimes \mid x\right\rangle\left\langle\left. x\right|_{X} .\right.
$$

In this case, we apply Lemma 31 to find that

$$
I_{\alpha}(A ; B \mid X)=\frac{\alpha}{\alpha-1} \log \sum_{x} p_{X}(x) \exp \left\{\left(\frac{\alpha-1}{\alpha}\right) I_{\alpha}(A ; B)_{\psi_{x} \otimes \phi_{x}}\right\} .
$$

The Rényi mutual information is equal to zero for any product state. This is easily seen from (3.15) and the fact that the Rényi relative entropy is non-negative. This implies that

$$
I_{\alpha}(A ; B \mid X)=\frac{\alpha}{\alpha-1} \log \sum_{x} p_{X}(x)=0 .
$$

Remark 9 Assuming [BSW15a, Conjecture 34], it follows from the faithfulness of the original von Neumann entropy-based squashed entanglement [BCY11] and Proposition 8 that the Rényi squashed entanglement $E_{\alpha}^{\mathrm{sq}}(A ; B)_{\rho}$ is faithful for $\alpha \in(1, \infty)$ (i.e., equals zero iff the state $\rho_{A B}$ is separable).

Proposition 10 On tensor-product states $\rho_{A_{1} A_{2} B_{1} B_{2}} \equiv \sigma_{A_{1} B_{1}} \otimes \tau_{A_{2} B_{2}} \in \mathcal{S}\left(\mathcal{H}_{A_{1} A_{2} B_{1} B_{2}}\right)$ the Rényi squashed entanglement is subadditive for $\alpha \in(0,1) \cup(1, \infty)$, in the sense that

$$
E_{\alpha}^{\mathrm{sq}}\left(A_{1} A_{2} ; B_{1} B_{2}\right)_{\rho} \leq E_{\alpha}^{\mathrm{sq}}\left(A_{1} ; B_{1}\right)_{\sigma}+E_{\alpha}^{\mathrm{sq}}\left(A_{2} ; B_{2}\right)_{\tau} .
$$

Proof. Let $\sigma_{A_{1} B_{1} E_{1}}$ be some extension of $\sigma_{A_{1} B_{1}}$, and let $\tau_{A_{2} B_{2} E_{2}}$ be some extension of $\tau_{A_{2} B_{2}}$. Then

$$
\omega_{A_{1} B_{1} E_{1} A_{2} B_{2} E_{2}} \equiv \sigma_{A_{1} B_{1} E_{1}} \otimes \tau_{A_{2} B_{2} E_{2}}
$$

is an extension of $\rho_{A_{1} A_{2} B_{1} B_{2}}$, so that

$$
\begin{aligned}
2 E_{\alpha}^{\mathrm{sq}}\left(A_{1} A_{2} ; B_{1} B_{2}\right)_{\rho} & \leq I_{\alpha}\left(A_{1} A_{2} ; B_{1} B_{2} \mid E_{1} E_{2}\right)_{\omega} \\
& =I_{\alpha}\left(A_{1} ; B_{1} \mid E_{1}\right)_{\sigma}+I_{\alpha}\left(A_{2} ; B_{2} \mid E_{2}\right)_{\tau} .
\end{aligned}
$$

The inequality is from the definition of Rényi squashed entanglement, and the equality follows by direct substitution into (3.18). Since the inequality is independent of the particular extensions of $\sigma_{A_{1} B_{1}}$ and $\tau_{A_{2} B_{2}}$, the statement of the proposition follows.

\subsection{Relations to Rényi entropy of entanglement and Rényi entanglement of formation}

The entropy of entanglement [BBPS96] and the entanglement of formation [BDSW96] are among the earliest proposed measures of entanglement for pure and mixed bipartite states, respectively. While the former is defined as the von Neumann entropy of either reduced density operators of a 
bipartite pure state, the latter is based upon the entropy of entanglement via an extended convex roof construction. The entanglement of formation of a bipartite state $\rho_{A B}$ is defined as

$$
E^{F}(A ; B)_{\rho} \equiv \min _{\left\{p_{X}(x),\left|\psi^{x}\right\rangle_{A B}\right\}}\left\{\sum_{x} p_{X}(x) E\left(\psi_{A B}^{x}\right): \rho_{A B}=\sum_{x} p_{X}(x)\left|\psi^{x}\right\rangle\left\langle\left.\psi^{x}\right|_{A B}\right\}\right.
$$

where $E\left(\psi_{A B}^{x}\right)=H(A)_{\psi^{x}}$ is the entropy of entanglement of pure state $\left|\psi^{x}\right\rangle_{A B}$. Note that the expression in (4.34) can be written more concisely in terms of the conditional entropy $H(A \mid X)_{\sigma}$, where $\sigma_{X A B}$ is a classical-quantum state of the following form:

$$
\sigma_{X A B} \equiv \sum_{x} p_{X}(x)|x\rangle\left\langle\left. x\right|_{X} \otimes \mid \psi^{x}\right\rangle\left\langle\left.\psi^{x}\right|_{A B}\right.
$$

That is,

$$
E^{F}(A ; B)_{\rho}=\min _{\left\{p_{X}(x),\left|\psi^{x}\right\rangle_{A B}\right\}}\left\{H(A \mid X)_{\sigma}: \rho_{A B}=\sum_{x} p_{X}(x)\left|\psi^{x}\right\rangle\left\langle\left.\psi^{x}\right|_{A B}\right\} .\right.
$$

We now consider a Rényi entropy of entanglement and a Rényi entanglement of formation defined based upon the Rényi entropy and the Rényi conditional entropy of (3.13), respectively. We also derive some relationships between the Rényi squashed entanglement and these quantities. While the Rényi entropy of entanglement of a pure state $\psi_{A B}^{x}$ is simply the Rényi entropy $H_{\alpha}(A)_{\psi}$, the Rényi entanglement of formation of a state $\rho_{A B}$ is defined as follows.

Definition 11 The Rényi entanglement of formation of $\rho_{A B} \in \mathcal{S}\left(\mathcal{H}_{A B}\right)$ is defined for $\alpha \in(0,1) \cup$ $(1, \infty)$ as

$$
E_{\alpha}^{F}(A ; B)_{\rho} \equiv \inf _{\left\{p_{X}(x),\left|\psi^{x}\right\rangle_{A B}\right\}}\left\{H_{\alpha}(A \mid X)_{\sigma}: \rho_{A B}=\sum_{x} p_{X}(x)\left|\psi^{x}\right\rangle\left\langle\left.\psi^{x}\right|_{A B}\right\},\right.
$$

where $\sigma_{X A B}$ is a classical-quantum state of the form as in (4.35).

Proposition 12 The Rényi entanglement of formation of $\rho_{A B} \in \mathcal{S}\left(\mathcal{H}_{A B}\right)$ can be written as

$E_{\alpha}^{F}(A ; B)_{\rho}=\min _{\left\{p_{X}(x),\left|\psi^{x}\right\rangle_{A B}\right.}\left\{\frac{\alpha}{1-\alpha} \log \sum_{x} p_{X}(x)\left[\operatorname{Tr}\left\{\left(\psi_{A}^{x}\right)^{\alpha}\right\}^{1 / \alpha}\right]: \rho_{A B}=\sum_{x} p_{X}(x)\left|\psi^{x}\right\rangle\left\langle\left.\psi^{x}\right|_{A B}\right\}\right.$.

Proof. By applying the Sibson identity mentioned in [SW13] to $H_{\alpha}(A \mid X)_{\sigma}$ in (4.37), we find that

$$
\begin{aligned}
H_{\alpha}(A \mid X)_{\sigma} & =\frac{\alpha}{1-\alpha} \log \operatorname{Tr}\left\{\left(\operatorname{Tr}_{A}\left\{\sigma_{A X}^{\alpha}\right\}\right)^{1 / \alpha}\right\} \\
& =\frac{\alpha}{1-\alpha} \log \operatorname{Tr}\left\{\left(\operatorname{Tr}_{A}\left\{\sum_{x} p_{X}^{\alpha}(x)|x\rangle\left\langle\left. x\right|_{X} \otimes\left(\psi_{A}^{x}\right)^{\alpha}\right\}\right)^{1 / \alpha}\right\}\right. \\
& =\frac{\alpha}{1-\alpha} \log \operatorname{Tr}\left\{\left(\left\{\sum_{x} p_{X}^{\alpha}(x)|x\rangle\left\langle\left. x\right|_{X} \operatorname{Tr}\left\{\left(\psi_{A}^{x}\right)^{\alpha}\right\}\right\}\right)^{1 / \alpha}\right\}\right. \\
& =\frac{\alpha}{1-\alpha} \log \sum_{x} p_{X}(x)\left[\operatorname{Tr}\left\{\left(\psi_{A}^{x}\right)^{\alpha}\right\}^{1 / \alpha}\right] .
\end{aligned}
$$


Finally, by an application of the Carathéodory theorem, we know that the infimum can be replaced by a minimum because $2^{\frac{1-\alpha}{\alpha} H_{\alpha}(A \mid X)_{\sigma}}$ can be written as a convex combination of $\operatorname{Tr}\left\{\left(\psi_{A}^{x}\right)^{\alpha}\right\}^{1 / \alpha}$, which is a continuous function of the marginals of the elements of a convex decomposition of the state $\rho_{A B}$ and these marginals are elements of a compact set.

Proposition 13 For a pure state $\phi_{A B} \in \mathcal{S}\left(\mathcal{H}_{A B}\right)$, the Rényi squashed entanglement is related to the Rényi entropy of entanglement as

$$
E_{\alpha}^{\mathrm{sq}}(A ; B)_{\phi}=H_{(2-\alpha) / \alpha}(A)_{\phi},
$$

for $\alpha \in(0,1) \cup(1,2]$.

Proof. Consider that any extension of a pure state $\phi_{A B}$ is of the form $\phi_{A B} \otimes \omega_{E}$. So applying Lemma 30, we find that

$$
I_{\alpha}(A ; B \mid E)_{\phi \otimes \omega}=I_{\alpha}(A ; B)_{\phi} .
$$

So now it is a matter of evaluating the Rényi mutual information of a pure state. Consider that

$$
\begin{aligned}
I_{\alpha}(A ; B)_{\phi} & =\frac{\alpha}{\alpha-1} \log \operatorname{Tr}\left\{\left(\operatorname{Tr}_{A}\left\{\phi_{A}^{1-\alpha} \phi_{A B}^{\alpha}\right\}\right)^{1 / \alpha}\right\} \\
& =\frac{\alpha}{\alpha-1} \log \operatorname{Tr}\left\{\left(\operatorname{Tr}_{A}\left\{\phi_{A}^{1-\alpha} \phi_{A B}\right\}\right)^{1 / \alpha}\right\} \\
& =\frac{\alpha}{\alpha-1} \log \operatorname{Tr}\left\{\left(\operatorname{Tr}_{A}\left\{\phi_{B}^{1-\alpha} \phi_{A B}\right\}\right)^{1 / \alpha}\right\} \\
& =\frac{\alpha}{\alpha-1} \log \operatorname{Tr}\left\{\left(\phi_{B}^{1-\alpha} \phi_{B}\right)^{1 / \alpha}\right\} \\
& =\frac{\alpha}{\alpha-1} \log \operatorname{Tr}\left\{\phi_{B}^{(2-\alpha) / \alpha}\right\} \\
& =2 \frac{1}{1-(2-\alpha) / \alpha} \log \operatorname{Tr}\left\{\phi_{B}^{(2-\alpha) / \alpha}\right\} \\
& =2 H_{(2-\alpha) / \alpha}(B)_{\phi} \\
& =2 H_{(2-\alpha) / \alpha}(A)_{\phi} .
\end{aligned}
$$

The first equality follows from applying (3.16). The second equality follows because $\phi_{A B}^{\alpha}=\phi_{A B}$ for a pure state $\phi$. The third equality follows because $\phi_{A}^{1-\alpha}|\phi\rangle_{A B}=\phi_{B}^{1-\alpha}|\phi\rangle_{A B}$ for a pure bipartite state $\phi$. The fourth equality follows by taking the partial trace over system $A$. The rest of the equalities are straightforward, by applying the definition of the Rényi entropy.

Corollary 14 The Rényi squashed entanglement is normalized on maximally entangled states, in the sense that for $\alpha \in(0,1) \cup(1,2]$

$$
E_{\alpha}^{\mathrm{sq}}(A ; B)_{\Phi}=\log d
$$

where $d$ is the Schmidt rank of the maximally entangled state $\Phi_{A B}$.

Proof. This follows from applying Proposition 13 and from the fact that the Rényi entropy is equal to $\log d$ for a maximally mixed state of dimension $d$. 


\begin{tabular}{|c|c|c|}
\hline Property & Squashed Ent. of (1.1) & Rényi Squashed Ent. of (4.1) \\
\hline \hline Normalized & $\checkmark$ & $\begin{array}{c}\checkmark \\
\text { for } \alpha \in(0,1) \cup(1,2]\end{array}$ \\
\hline LOCC Monotonicity & $\checkmark$ & $\begin{array}{c}\text { if Conj. 1 true, } \\
\text { then true for } \alpha \in(0,1) \cup(1,2]\end{array}$ \\
\hline Convexity & $\checkmark$ & $\begin{array}{c}\checkmark \\
\text { for } \alpha \in(0,1)\end{array}$ \\
\hline Faithfulness & $\checkmark$ & $\begin{array}{c}\text { vanishing on sep. states for } \alpha \in(0,1) \cup(1, \infty) ; \\
\text { if [BSW15a, Conj. 34] true, } \\
\text { then true for } \alpha \in(1, \infty)\end{array}$ \\
\hline Additivity & & subadditive for $\alpha \in(0,1) \cup(1, \infty)$ \\
\hline Monogamy & $\checkmark$ & $?$ \\
\hline Non-lockability & $\checkmark$ & $?$ \\
\hline Asymptotic Continuity & $\checkmark$ & $?$ \\
\hline
\end{tabular}

Table 1: Properties of the Rényi squashed entanglement in comparison to those of the original von Neumann entropy based squashed entanglement. The question marks indicate properties that remain open for the Rényi squashed entanglement of (4.1). Two of the properties for the Rényi squashed entanglement rely on conjectures. Conj. 1 is the statement that the Rényi conditional quantum mutual information obeys monotonicity with respect to quantum operations on system $A$, i.e., for $\rho_{A B E} \in \mathcal{S}\left(\mathcal{H}_{A B E}\right)$ and $\alpha \in(0,1) \cup(1,2], I_{\alpha}(A ; B \mid E)_{\rho} \geq I_{\alpha}\left(A^{\prime} ; B \mid E\right)_{\tau}$, where $\tau_{A^{\prime} B E} \equiv$ $\mathcal{M}_{A \rightarrow A^{\prime}}\left(\rho_{A B E}\right)$ and $\mathcal{M}_{A \rightarrow A^{\prime}}$ is a CPTP map acting on system $A$ alone. [BSW15a, Conj. 34] is the statement that the Rényi conditional quantum mutual information is monotone non-decreasing in the Rényi parameter, i.e., $I_{\alpha}(A ; B \mid C) \leq I_{\beta}(A ; B \mid C)$ for $0 \leq \alpha \leq \beta$. 
Proposition 15 For $\rho_{A B} \in \mathcal{S}\left(\mathcal{H}_{A B}\right)$, the Rényi squashed entanglement is bounded from above by the Rényi entanglement of formation for $\alpha \in(0,1) \cup(1,2)$ as

$$
E_{(2-\alpha) / \alpha}^{F}(A ; B)_{\rho} \geq E_{\alpha}^{\mathrm{sq}}(A ; B)_{\rho} .
$$

Proof. Consider an ensemble $\left\{p_{X}(x),\left|\psi^{x}\right\rangle_{A B}\right\}$ which realizes $\rho_{A B}$, in the sense that

$$
\rho_{A B}=\sum_{x} p_{X}(x)\left|\psi^{x}\right\rangle\left\langle\left.\psi^{x}\right|_{A B}\right.
$$

We then form the classical-quantum state $\sigma_{X A B}$ :

$$
\sigma_{X A B}=\sum_{x} p_{X}(x)|x\rangle\left\langle\left. x\right|_{X} \otimes \mid \psi^{x}\right\rangle\left\langle\left.\psi^{x}\right|_{A B}\right.
$$

Consider that

$$
\begin{aligned}
H_{\beta}(A \mid X) & =\frac{\beta}{1-\beta} \log \sum_{x} p_{X}(x)\left[\operatorname{Tr}\left\{\left(\psi_{A}^{x}\right)^{\beta}\right\}^{1 / \beta}\right] \\
& =\frac{1}{1-\beta} \log \left[\sum_{x} p_{X}(x)\left[\operatorname{Tr}\left\{\left(\psi_{A}^{x}\right)^{\beta}\right\}^{1 / \beta}\right]\right]^{\beta} .
\end{aligned}
$$

For $\beta \in(0,1) \cup(1, \infty)$, we have from concavity / convexity of the function $x^{\beta}$ that

$$
H_{\beta}(A \mid X) \geq \frac{1}{1-\beta} \log \sum_{x} p_{X}(x) \operatorname{Tr}\left\{\left(\psi_{A}^{x}\right)^{\beta}\right\} .
$$

Let $\beta=(2-\alpha) / \alpha$. Then, from (4.59), for $\alpha \in(0,1) \cup(1,2)$, we have that

$$
H_{(2-\alpha) / \alpha}(A \mid X) \geq \frac{1}{1-(2-\alpha) / \alpha} \log \sum_{x} p_{X}(x) \operatorname{Tr}\left\{\left(\psi_{A}^{x}\right)^{(2-\alpha) / \alpha}\right\} .
$$

Now consider that

$$
\begin{aligned}
\frac{1}{2} I_{\alpha}(A ; B \mid X)_{\sigma} & =\frac{1}{2} \frac{\alpha}{\alpha-1} \log \sum_{x} p_{X}(x) 2^{\left(\frac{\alpha-1}{\alpha}\right) I_{\alpha}(A ; B)_{\psi^{x}}} \\
& =\frac{1}{2} \frac{\alpha}{\alpha-1} \log \sum_{x} p_{X}(x) 2^{\left(\frac{\alpha-1}{\alpha}\right) \frac{\alpha}{\alpha-1} \log \operatorname{Tr}\left\{\left(\psi_{A}^{x}\right)^{(2-\alpha) / \alpha}\right\}} \\
& =\frac{1}{2} \frac{\alpha}{\alpha-1} \log \sum_{x} p_{X}(x) \operatorname{Tr}\left\{\left(\psi_{A}^{x}\right)^{(2-\alpha) / \alpha}\right\} \\
& =\frac{1}{1-(2-\alpha) / \alpha} \log \sum_{x} p_{X}(x) \operatorname{Tr}\left\{\left(\psi_{A}^{x}\right)^{(2-\alpha) / \alpha}\right\}
\end{aligned}
$$

The first equality follows by applying Lemma [31. The second equality follows from some steps given in the proof of Proposition [13. The last two equalities are straightforward.

From (4.60) and (4.64), we have that

$$
H_{(2-\alpha) / \alpha}(A \mid X) \geq \frac{1}{2} I_{\alpha}(A ; B \mid X) .
$$


The statement of the proposition follows from (4.65), because

$$
\frac{1}{2} I_{\alpha}(A ; B \mid X) \geq E_{\alpha}^{\mathrm{sq}}(A ; B)_{\rho},
$$

which in turn follows because the state in (4.56) is a particular extension of the state $\rho_{A B}$ and by definition, the Rényi squashed entanglement is equal to the infimum over all such extensions. So putting together (4.65) and (4.66) gives the statement of the proposition.

\section{Rényi Quantum Discord}

In this section, we define a Rényi quantum discord and explore some properties of it. We also give an expression for the Rényi discord of pure bipartite states. Further, using the Rényi discord, we discuss an operational characterization of quantum states with small von Neumann entropy-based discord, which follows from [BSW15a, Conjecture 34].

Definition 16 The Rényi quantum discord of $\rho_{A B} \in \mathcal{S}(\mathcal{H})$ is defined for $\alpha \in(0,1) \cup(1,2]$ as

$$
D^{\alpha}(\bar{A} ; B)_{\rho} \equiv \inf _{\left\{\Lambda_{x}\right\}} I_{\alpha}(E ; B \mid X)_{\omega},
$$

where

$$
\omega_{E X B} \equiv U_{A \rightarrow E X} \rho_{A B} U_{A \rightarrow E X}^{\dagger},
$$

with $U_{A \rightarrow E X}$ being an isometric extension of the measurement map $\sigma \rightarrow \sum_{x} \operatorname{Tr}\left\{\Lambda_{x} \sigma\right\}|x\rangle\left\langle\left. x\right|_{X}\right.$ acting on system $A$.

\subsection{Properties of the Rényi discord}

We now prove that the Rényi quantum discord for $\alpha \in(0,1) \cup(1,2]$ is non-negative, is invariant with respect to local unitaries, vanishes on the set of classical-quantum states, and is optimized by a rank-one POVM.

Proposition 17 The Rényi quantum discord $D^{\alpha}(\bar{A} ; B)_{\rho}$ is non-negative for $\alpha \in(0,1) \cup(1,2]$.

Proof. This follows easily from the fact that the Rényi conditional mutual information is nonnegative for $\alpha \in(0,1) \cup(1,2]$ [BSW15a, Corollary 16].

Proposition 18 For a classical-quantum state $\rho_{A B} \in \mathcal{S}\left(\mathcal{H}_{A B}\right)$ the Rényi quantum discord is equal to zero for $\alpha \in(0,1) \cup(1, \infty)$.

Proof. Consider a classical-quantum state $\rho_{A B}$ :

$$
\rho_{A B}=\sum_{x} p_{X}(x)|x\rangle\left\langle\left. x\right|_{A} \otimes \rho_{B}^{x} .\right.
$$

Let the dilation of a von Neumann measurement of system $A$ be $|x\rangle_{A} \rightarrow|x\rangle_{X}|x\rangle_{E}$, so that it produces

$$
\rho_{X E B} \equiv \sum_{x} p(x)|x\rangle\left\langle\left. x\right|_{X} \otimes \mid x\right\rangle\left\langle\left. x\right|_{E} \otimes \rho_{B}^{x}\right.
$$


So the conditioning system $X$ is classical. Applying Lemma 31, we find that

$$
I_{\alpha}(E ; B \mid X)=\frac{\alpha}{\alpha-1} \log \sum_{x} p_{X}(x) 2^{\left(\frac{\alpha-1}{\alpha}\right) I_{\alpha}(E ; B)|x\rangle\langle x| \otimes \rho^{x}}=\frac{\alpha}{\alpha-1} \log \sum_{x} p_{X}(x)=0 .
$$

Combining this with the previous proposition, we see that it is equal to zero for classical-quantum states.

Proposition 19 The Rényi quantum discord $D^{\alpha}(\bar{A} ; B)_{\rho}$ is invariant with respect to local unitaries for $\alpha \in(0,1) \cup(1, \infty)$.

Proof. First, from the definition of $I_{\alpha}(A ; B \mid E)$, we can see that this quantity is invariant with respect to local unitaries. It then immediately follows that the Rényi discord is invariant with respect to local unitaries $U_{A} \otimes V_{B}$. That is, if the optimal measurement is given by $\left\{\Lambda_{A}^{x}\right\}$, then the optimal measurement with respect to a local unitary $U_{A}$ can be taken as $\left\{U_{A}^{\dagger} \Lambda_{A}^{x} U_{A}\right\}$.

Proposition 20 It suffices to optimize the Rényi quantum discord (Definition 16) with respect to rank-one POVMs for $\alpha \in(0,1) \cup(1,2]$.

Proof. Consider an arbitrary measurement map

$$
\mathcal{M}(\sigma) \equiv \sum_{x} \operatorname{Tr}\left\{\Lambda_{x} \sigma\right\}|x\rangle\left\langle\left. x\right|_{X},\right.
$$

and a spectral decomposition of each $\Lambda_{x}$ :

$$
\Lambda_{x}=\sum_{y} \mu_{x y}\left|\phi_{x, y}\right\rangle\left\langle\phi_{x, y}\right| .
$$

For a fixed $x$, the set $\left\{\left|\phi_{x, y}\right\rangle\right\}_{y}$ is orthonormal. Furthermore, the set $\left\{\mu_{x y}\left|\phi_{x, y}\right\rangle\left\langle\phi_{x, y}\right|\right\}_{x, y}$ forms a POVM (a rank-one refinement of the original POVM). We can then rewrite the measurement map as

$$
\mathcal{M}(\sigma)=\sum_{x, y} \operatorname{Tr}\left\{\mu_{x y}\left|\phi_{x, y}\right\rangle\left\langle\phi_{x, y}\right| \sigma\right\}|x\rangle\left\langle\left. x\right|_{X} .\right.
$$

In this way, we can already see that there is some loss of information when discarding the outcome $y$. An isometric extension of the original measurement map is specified by

$$
U_{A \rightarrow E X_{E} Y X}^{\mathcal{M}}|\psi\rangle_{A} \equiv \sum_{x, y} \sqrt{\mu_{x y}}\left(\left|\phi_{x, y}\right\rangle_{E}\left\langle\left.\phi_{x, y}\right|_{A}\right)|\psi\rangle_{A} \otimes|x\rangle_{X_{E}}|y\rangle_{Y} \otimes|x\rangle_{X} .\right.
$$

This is because the original map is recovered by tracing over the environmental systems $E X_{E} Y$ :

$$
\begin{aligned}
& \operatorname{Tr}_{E X_{E} Y}\left\{U_{A \rightarrow E X_{E} Y X^{\mathcal{M}}}^{\mathcal{M}} \sigma\left(U_{A \rightarrow E X_{E} Y X}^{\mathcal{M}}\right)^{\dagger}\right\} \\
& =\operatorname{Tr}_{E X_{E} Y}\left\{\sum _ { x , y , x ^ { \prime } , y ^ { \prime } } \sqrt { \mu _ { x y } } \sqrt { \mu _ { x ^ { \prime } y ^ { \prime } } } \left(\left|\phi_{x, y}\right\rangle_{E}\left\langle\left.\phi_{x, y}\right|_{A}\right) \sigma\left|\phi_{x^{\prime}, y^{\prime}}\right\rangle_{A}\left\langle\left.\phi_{x^{\prime}, y^{\prime}}\right|_{E} \otimes \mid x\right\rangle\left\langle\left. x^{\prime}\right|_{X_{E}} \otimes \mid y\right\rangle\left\langle\left. y^{\prime}\right|_{Y} \otimes \mid x\right\rangle\left\langle\left. x^{\prime}\right|_{X}\right\}\right.\right. \\
& =\sum_{x, y} \mu_{x y}\left(\langle \phi _ { x , y } | _ { E } | \phi _ { x , y } \rangle _ { E } \langle \phi _ { x , y } | _ { A } ) \sigma | \phi _ { x , y } \rangle _ { A } \otimes | x \rangle \left\langle\left.x\right|_{X}\right.\right. \\
& =\sum_{x, y} \operatorname{Tr}\left\{\mu_{x y}\left|\phi_{x, y}\right\rangle\left\langle\phi_{x, y}\right| \sigma\right\}|x\rangle\left\langle\left. x\right|_{X}\right. \\
& =\mathcal{M}(\sigma)
\end{aligned}
$$


Let $\psi_{R A B}^{\rho}$ be a purification of the state $\rho_{A B}$ on which we are evaluating the Rényi discord. Then let $\omega_{R E X_{E} Y X B}$ be the following pure state:

$$
\omega_{R E X_{E} Y X B} \equiv U_{A \rightarrow E X_{E} Y X}^{\mathcal{M}} \psi_{R A B}^{\rho}\left(U_{A \rightarrow E X_{E} Y X}^{\mathcal{M}}\right)^{\dagger} .
$$

Consider the following chain of inequalities:

$$
I_{\alpha}\left(E X_{E} Y ; B \mid X\right)_{\omega}=I_{\alpha}\left(B ; E X_{E} Y \mid R\right)_{\omega} \geq I_{\alpha}\left(B ; E X_{E} \mid R\right)_{\omega}=I_{\alpha}\left(E X_{E} ; B \mid X Y\right)_{\omega} .
$$

The first equality is by duality of (3.20). The second inequality is from monotonicity with respect to local quantum operation. The last equality is again from duality. But now consider that the last quantity corresponds to the quantum discord for the following refined rank-one measurement map:

$$
\sigma \rightarrow \sum_{x, y} \operatorname{Tr}\left\{\mu_{x y}\left|\phi_{x, y}\right\rangle\left\langle\phi_{x, y}\right| \sigma\right\}|x\rangle\left\langle\left. x\right|_{X} \otimes \mid y\right\rangle\left\langle\left. y\right|_{Y}\right.
$$

Furthermore, the systems $E X_{E}$ above play the role of the environment of the refined rank-one measurement map from $A$ to $X$ and $Y$. This is because

$$
\begin{aligned}
& \operatorname{Tr}_{E X_{E}}\left\{U_{A \rightarrow E X_{E} Y X^{\mathcal{M}}}^{\mathcal{M}} \sigma\left(U_{A \rightarrow E X_{E} Y X}^{\mathcal{M}}\right)^{\dagger}\right\} \\
& =\operatorname{Tr}_{E X_{E}}\left\{\sum _ { x , y , x ^ { \prime } , y ^ { \prime } } \sqrt { \mu _ { x y } } \sqrt { \mu _ { x ^ { \prime } y ^ { \prime } } } \left(\left|\phi_{x, y}\right\rangle_{E}\left\langle\left.\phi_{x, y}\right|_{A}\right) \sigma\left|\phi_{x^{\prime}, y^{\prime}}\right\rangle_{A}\left\langle\left.\phi_{x^{\prime}, y^{\prime}}\right|_{E} \otimes \mid x\right\rangle\left\langle\left. x^{\prime}\right|_{X_{E}} \otimes \mid y\right\rangle\left\langle\left. y^{\prime}\right|_{Y} \otimes \mid x\right\rangle\left\langle\left. x^{\prime}\right|_{X}\right\}\right.\right. \\
& =\sum_{x, y, y^{\prime}} \mu_{x y}\left(\langle \phi _ { x , y ^ { \prime } } | _ { E } | \phi _ { x , y } \rangle _ { E } \langle \phi _ { x , y } | _ { A } ) \sigma | \phi _ { x , y ^ { \prime } } \rangle _ { A } \otimes | y \rangle \langle y ^ { \prime } | _ { Y } \otimes | x \rangle \left\langle\left.x\right|_{X}\right.\right. \\
& =\sum_{x, y, y^{\prime}} \mu_{x y}\left\langle\left.\phi_{x, y}\right|_{A} \sigma \mid \phi_{x, y}\right\rangle_{A} \otimes|y\rangle\left\langle\left. y\right|_{Y} \otimes \mid x\right\rangle\left\langle\left. x\right|_{X}\right. \\
& =\sum_{x, y} \operatorname{Tr}\left\{\mu_{x y}\left|\phi_{x, y}\right\rangle\left\langle\phi_{x, y}\right| \sigma\right\}|x\rangle\left\langle\left. x\right|_{X} \otimes \mid y\right\rangle\left\langle\left. y\right|_{Y}\right.
\end{aligned}
$$

Since a rank-one POVM always achieves a lower value of the Rényi quantum discord, it suffices to optimize over only these kinds of POVMs when calculating it.

At this point we would like to note that a different Rényi generalization of the quantum discord, proposed in $\left[\mathrm{MBP}^{+} 15\right]$, is optimized by a rank-one POVM as well. This quantity was defined based upon an optimization over rank-one projective measurements, but can be extended to an optimization over all POVMs. Consider the definition for the Rényi discord of a bipartite state $\rho_{A B}$ proposed in $\left[\mathrm{MBP}^{+} 15\right.$, Eq. (26)] (with projective measurements replaced by POVMs $\left\{\Lambda^{x}\right\}$ ):

$$
\mathfrak{D}^{\alpha}(\bar{A} ; B)_{\rho} \equiv \min _{\sigma_{A}, \sigma_{B}} D_{\alpha}\left(\rho_{A B} \| \sigma_{A} \otimes \sigma_{B}\right)-\max _{\left\{\Lambda^{x}\right\}} \min _{\sigma_{X}, \sigma_{B}} D_{\alpha}\left(\rho_{X B} \| \sigma_{X} \otimes \sigma_{B}\right) .
$$

The sufficiency of rank-one POVMs $\left\{\Lambda^{x}\right\}$ in the above definition can be proven along the following lines. Similar to the proof of Proposition 20, consider a spectral decomposition of the POVM elements $\left\{\Lambda^{x}\right\}$ :

$$
\Lambda_{x}=\sum_{y} \mu_{x y}\left|\phi_{x, y}\right\rangle\left\langle\phi_{x, y}\right| .
$$




\begin{tabular}{|c|c|c|c|}
\hline Property & Discord of (1.1) & Rényi Discord of (5.1) & $\begin{array}{c}\text { Rényi Discord of (5.21) } \\
\text { as in [MBP } \text { MB }^{+} \text {] }\end{array}$ \\
\hline \hline Non-negative & $\checkmark$ & $\begin{array}{c}\checkmark \\
\text { for } \alpha \in(0,1) \cup(1,2]\end{array}$ & $\begin{array}{c}\checkmark \\
\text { for } \alpha \in[1 / 2,1) \cup(1, \infty)\end{array}$ \\
\hline Vanishing on cq-states & $\checkmark$ & $\begin{array}{c}\checkmark \\
\text { for } \alpha \in(0,1) \cup(1, \infty)\end{array}$ & for $\alpha \in(0,1) \cup(1, \infty)$ \\
\hline Unitary invariance & $\checkmark$ & $\begin{array}{c}\checkmark \\
\text { for } \alpha \in(0,1) \cup(1, \infty)\end{array}$ & for $\alpha \in(0,1) \cup(1, \infty)$ \\
\hline Rank-1 POVM optimal & $\checkmark$ & $\begin{array}{c}\checkmark \\
\text { for } \alpha \in(0,1) \cup(1,2]\end{array}$ & for $\alpha \in[1 / 2,1) \cup(1, \infty)$ \\
\hline Monotone in $\alpha$ & N/A & $\begin{array}{c}\text { if BSW15a, Conj. 34] } \\
\text { true, then true }\end{array}$ & $\boldsymbol{X}$ \\
\hline
\end{tabular}

Table 2: Properties of the Rényi quantum discord of (5.1) in comparison to those of the original von Neumann entropy based quantum discord and the Rényi quantum discord proposed in $\mathrm{MBP}^{+} 15$, Eq. (26)]. In the first column, cq-states refers to classical-quantum states. The property of monotonicity in $\alpha$ for the Rényi quantum discord of (5.1) relies on [BSW15a, Conj. 34], which is the statement that the Rényi conditional quantum mutual information is monotone non-decreasing in the Rényi parameter, i.e., $I_{\alpha}(A ; B \mid C) \leq I_{\beta}(A ; B \mid C)$ for $0 \leq \alpha \leq \beta$. The Rényi discord of $\left.\mathrm{MBP}^{+} 15\right]$ is not monotone in $\alpha$ because it is equal to a difference of two Rényi relative entropies.

Once again, for a fixed $x$, the set $\left\{\left|\phi_{x, y}\right\rangle\right\}_{y}$ is orthonormal, and the set $\left\{\mu_{x y}\left|\phi_{x, y}\right\rangle\left\langle\phi_{x, y}\right|\right\}_{x, y}$ forms a rank-one refinement of the original POVM. The output state corresponding to the measurement consisting of these above rank-one refinements can be written as

$$
\rho_{X Y B}=\sum_{x, y}|x\rangle\left\langle\left. x\right|_{X} \otimes \mid y\right\rangle\left\langle\left. y\right|_{Y} \otimes \mu_{x y}\left\langle\left.\phi_{x, y}\right|_{A} \rho_{A B} \mid \phi_{x, y}\right\rangle_{A} .\right.
$$

Due to the monotonicity of the Rényi relative entropy with respect to local quantum operations, we have that

$$
\min _{\sigma_{X Y}, \sigma_{B}} D_{\alpha}\left(\rho_{X Y B} \| \sigma_{X Y} \otimes \sigma_{B}\right) \geq \min _{\sigma_{X}, \sigma_{B}} D_{\alpha}\left(\rho_{X B} \| \sigma_{X} \otimes \sigma_{B}\right)
$$

This implies

$$
\max _{\left\{\mu_{y}\left|\phi_{x, y}\right\rangle\left\langle\phi_{x, y}\right|\right\}_{x, y}} \min _{\sigma_{X Y}, \sigma_{B}} D_{\alpha}\left(\rho_{X Y B} \| \sigma_{X Y} \otimes \sigma_{B}\right) \geq \max _{\left\{\mu_{y}\left|\phi_{x, y}\right\rangle\left\langle\phi_{x, y}\right|\right\}_{x, y}} \min _{\sigma_{X}, \sigma_{B}} D_{\alpha}\left(\rho_{X B} \| \sigma_{X} \otimes \sigma_{B}\right) .
$$

Hence, the second term in (5.21) can be replaced with

$$
\max _{\left\{\mu_{y}\left|\phi_{x, y}\right\rangle\left\langle\phi_{x, y}\right|\right\}_{x, y}} \min _{\sigma_{X Y}, \sigma_{B}} D_{\alpha}\left(\rho_{X Y B} \| \sigma_{X Y} \otimes \sigma_{B}\right) .
$$

Therefore, it suffices to optimize the Rényi quantum discord defined in $\left[\mathrm{MBP}^{+} 15\right.$, Eq. (26)] over only rank-one POVMs.

\subsection{Rényi quantum discord for pure bipartite states}

We now give an expression for the Rényi discord of pure bipartite states. 
Proposition 21 The Rényi quantum discord of a pure bipartite state $\psi_{A B} \in \mathcal{S}\left(\mathcal{H}_{A B}\right)$ for $\alpha \in$ $(0,1) \cup(1,2]$ is given by

$$
D^{\alpha}(\bar{A} ; B)_{\psi}=\inf _{\left\{\left|\varphi_{x}\right\rangle: \sum_{x}\left|\varphi_{x}\right\rangle\left\langle\varphi_{x}\right|=I\right\}} \frac{\alpha}{\alpha-1} \log \sum_{x} p(x)\left\langle\left.\xi_{x}\right|_{B} \psi_{B}^{1-\alpha} \mid \xi_{x}\right\rangle^{1 / \alpha}
$$

where

$$
\left|\xi_{x}\right\rangle_{B}=\frac{\left\langle\left.\varphi_{x}\right|_{A} \mid \psi\right\rangle_{A B}}{\sqrt{p(x)}}, \quad p(x)=\left\|\left\langle\left.\varphi_{x}\right|_{A} \mid \psi\right\rangle_{A B}\right\|_{2}^{2}
$$

and $\left\{\left|\varphi_{x}\right\rangle: \sum_{x}\left|\varphi_{x}\right\rangle\left\langle\varphi_{x}\right|=I\right\}$ denotes a rank-one POVM acting on system $A$.

Proof. We begin by recalling Proposition 20, i.e., that it suffices to optimize the Rényi quantum discord over rank-one POVMs. Let $\left\{\left|\varphi_{x}\right\rangle\left\langle\left.\varphi_{x}\right|_{A}\right\}_{x}\right.$ denote such a POVM, so that $\sum_{x}\left|\varphi_{x}\right\rangle\left\langle\left.\varphi_{x}\right|_{A}=I_{A}\right.$. Consider a bipartite pure state

$$
|\psi\rangle_{A B}=\sum_{y=0}^{d-1} \sqrt{\lambda(y)}\left|\tilde{\psi}_{y}\right\rangle_{A}\left|\psi_{y}\right\rangle_{B},
$$

where $\left|\tilde{\psi}_{y}\right\rangle$ and $\left|\psi_{y}\right\rangle$ are orthonormal bases in $\mathcal{H}_{A}$ and $\mathcal{H}_{B}$. The post measurement tripartite state is given by

$$
\omega_{B E X} \equiv U_{A \rightarrow E X} \psi_{A B} U_{A \rightarrow E X}^{\dagger},
$$

where $U_{A \rightarrow E X}$ is an isometric extension of the aforementioned rank-one measurement:

$$
U_{A \rightarrow E X} \equiv \sum_{x}|x\rangle_{E}|x\rangle_{X}\left\langle\left.\varphi_{x}\right|_{A} .\right.
$$

The above state, and the reduced states on systems $B E$ and $B$ can thus be equivalently written as

$$
\begin{aligned}
\omega_{B E X} & \equiv \sum_{x, y} \sqrt{p(x) p(y)}|x\rangle\left\langle\left. y\right|_{X} \otimes \mid x\right\rangle\left\langle\left. y\right|_{E} \otimes \mid \xi_{x}\right\rangle\left\langle\left.\xi_{y}\right|_{B},\right. \\
\omega_{B E} & \equiv \sum_{x} p(x)|x\rangle\left\langle\left. x\right|_{E} \otimes \mid \xi_{x}\right\rangle\left\langle\left.\xi_{x}\right|_{B},\right.
\end{aligned}
$$

where $p(x)=\operatorname{Tr}\left\{\left|\varphi_{x}\right\rangle\left\langle\left.\varphi_{x}\right|_{A} \mid \psi\right\rangle\left\langle\left.\psi\right|_{A B}\right\}\right.$ and $\left|\xi_{x}\right\rangle\left\langle\left.\xi_{x}\right|_{B}=\operatorname{Tr}_{A}\left\{\left|\varphi_{x}\right\rangle\left\langle\left.\varphi_{x}\right|_{A} \mid \psi\right\rangle\left\langle\left.\psi\right|_{A B}\right\} / p(x)\right.\right.$. The Rényi conditional quantum mutual information of $\omega_{B E X}$ is thereby given by

$$
\begin{aligned}
I_{\alpha}(E ; B \mid X)_{\omega} & =I_{\alpha}(B ; E)_{\omega} \\
& =\frac{\alpha}{\alpha-1} \log \operatorname{Tr}\left\{\operatorname{Tr}_{B}\left\{\omega_{B E}^{\alpha} \omega_{B}^{1-\alpha}\right\}^{1 / \alpha}\right\} \\
& =\frac{\alpha}{\alpha-1} \log \operatorname{Tr}\left\{\operatorname{Tr}_{B}\left\{\left(\sum_{x} p(x)^{\alpha}|x\rangle\left\langle\left. x\right|_{E} \otimes \mid \xi_{x}\right\rangle\left\langle\left.\xi_{x}\right|_{B}\right) \omega_{B}^{1-\alpha}\right\}^{1 / \alpha}\right\}\right. \\
& =\frac{\alpha}{\alpha-1} \log \operatorname{Tr}\left\{\sum _ { x } p ( x ) | x \rangle \left\langle\left.x\right|_{E} \operatorname{Tr}_{B}\left\{\left|\xi_{x}\right\rangle\left\langle\left.\xi_{x}\right|_{B} \omega_{B}^{1-\alpha}\right\}^{1 / \alpha}\right\}\right.\right. \\
& =\frac{\alpha}{\alpha-1} \log \operatorname{Tr}\left\{\sum_{x} p(x) \operatorname{Tr}_{B}\left\{\left|\xi_{x}\right\rangle\left\langle\left.\xi_{x}\right|_{B} \omega_{B}^{1-\alpha}\right\}^{1 / \alpha}\right\}\right. \\
& =\frac{\alpha}{\alpha-1} \log \sum_{x} p(x)\left\langle\left.\xi_{x}\right|_{B} \omega_{B}^{1-\alpha} \mid \xi_{x}\right\rangle^{1 / \alpha} .
\end{aligned}
$$


The first equality follows from application of duality of (3.20) along with the fact that $\omega_{B E X}$ is a pure state. The second and third equalities follow from (3.16), the fact that the system $E$ is classical, and the fact that the post-measurement states on system $B$ are pure whenever a rank-one POVM is performed on system $A$. The fourth one follows from tracing over the $E$ system. The fifth and sixth equalities are straightforward.

Corollary 22 The Rényi quantum discord of a maximally entangled state $\Phi_{A B} \in \mathcal{S}\left(\mathcal{H}_{A B}\right)$ simplifies to

$$
D^{\alpha}(\bar{A} ; B)_{\Phi}=\log |A| .
$$

Proof. For a maximally entangled state, $p(x)$ in (5.32) is equal to $1 /|A|$, and the reduced state on system $B$ is maximally mixed. The result then follows from (5.39).

\subsection{Conjectured remainder terms for non-negativity of quantum discord}

In [BSW15a, Conjecture 34], it was conjectured that a Rényi conditional quantum mutual information defined based upon the sandwiched Rényi relative entropy of (3.12) is also monotone in the Rényi parameter. That is, for a tripartite state $\rho_{A B C}$ and $0 \leq \alpha \leq \beta$, it was conjectured that

$$
\widetilde{I}_{\alpha}(A ; B \mid C)_{\rho} \leq \widetilde{I}_{\beta}(A ; B \mid C)_{\rho},
$$

where the "sandwiched" Rényi conditional mutual information is defined as [BSW15a, Section 6]

$$
\widetilde{I}_{\alpha}(A ; B \mid C)_{\rho} \equiv \frac{1}{\alpha-1} \log \left\|\rho_{A B C}^{1 / 2} \rho_{A C}^{(1-\alpha) / 2 \alpha} \rho_{C}^{(\alpha-1) / 2 \alpha} \rho_{B C}^{(1-\alpha) / 2 \alpha}\right\|_{2 \alpha}^{2 \alpha} .
$$

Proofs were given for this conjectured inequality in [BSW15a, Section 8] in some special cases, e.g., when the Rényi parameter $\alpha$ is in a neighborhood of one, and when $1 / \alpha+1 / \beta=2$. Furthermore, implications of the conjectured inequality for understanding states with small conditional quantum mutual information were discussed. In particular, it was pointed out that the following lower bounds on the conditional quantum mutual information hold as consequences of the conjectured inequality in (5.41):

$$
\begin{aligned}
& I(A ; B \mid C)_{\rho} \geq-\log F\left(\rho_{A B C}, \mathcal{R}_{C \rightarrow A C}^{P}\left(\rho_{B C}\right)\right), \\
& I(A ; B \mid C)_{\rho} \geq-\log F\left(\rho_{A B C}, \mathcal{R}_{C \rightarrow B C}^{P}\left(\rho_{A C}\right)\right),
\end{aligned}
$$

where $\mathcal{R}_{C \rightarrow A C}^{P}$ and $\mathcal{R}_{C \rightarrow B C}^{P}$ are Petz recovery maps [HJPW04]:

$$
\begin{aligned}
& \mathcal{R}_{C \rightarrow A C}^{P}(\cdot) \equiv \rho_{A C}^{1 / 2} \rho_{C}^{-1 / 2}(\cdot) \rho_{C}^{-1 / 2} \rho_{A C}^{1 / 2}, \\
& \mathcal{R}_{C \rightarrow B C}^{P}(\cdot) \equiv \rho_{B C}^{1 / 2} \rho_{C}^{-1 / 2}(\cdot) \rho_{C}^{-1 / 2} \rho_{B C}^{1 / 2} .
\end{aligned}
$$

Since the quantum discord is based upon the conditional quantum mutual information, we now examine the implications of this conjecture for the quantum discord, by writing down a corresponding lower bound on it. This lower bound if true would provide a characterization for states with small quantum discord (the von Neumann entropy based quantity that is). It has the interpretation of quantifying how far a quantum state is from being a fixed point of an entanglement-breaking channel. In case the discord is equal to zero we can conclude that the state is a fixed point of an entanglement-breaking channel. In this case, we can apply [FNW15, Theorem 5.3] to conclude the known result that any zero-discord state is in fact a classical-quantum state. 
Consequence 23 Assuming [BSW15a, Conjecture 34], the following lower bound holds for the quantum discord of $\rho_{A B} \in \mathcal{S}\left(\mathcal{H}_{A B}\right)$ :

$$
D(\bar{A} ; B)_{\rho} \geq \min _{\left\{\left|\phi_{x}\right\rangle: \sum_{x}\left|\phi_{x}\right\rangle\left\langle\phi_{x}\right|=I\right\}}-\log F\left(\rho_{A B}, \mathcal{E}_{A}\left(\rho_{A B}\right)\right),
$$

where

$$
\rho_{B}^{x} \equiv \frac{1}{\operatorname{Tr}\left\{\left|\phi_{x}\right\rangle\left\langle\left.\phi_{x}\right|_{A} \rho_{A B}\right\}\right.}\left\langle\left.\phi_{x}\right|_{A} \rho_{A B} \mid \phi_{x}\right\rangle_{A},
$$

and $\mathcal{E}_{A}$ is the following entanglement-breaking channel:

$$
\mathcal{E}_{A}\left(\sigma_{A}\right) \equiv \sum_{x}\left\langle\left.\phi_{x}\right|_{A} \sigma_{A} \mid \phi_{x}\right\rangle_{A} \frac{\rho_{A}^{1 / 2}\left|\phi_{x}\right\rangle\left\langle\left.\phi_{x}\right|_{A} \rho_{A}^{1 / 2}\right.}{\operatorname{Tr}\left\{\left|\phi_{x}\right\rangle\left\langle\left.\phi_{x}\right|_{A} \rho_{A}\right\}\right.} .
$$

Proof. Consider a rank-one measurement $\left\{\left|\phi_{x}\right\rangle\left\langle\phi_{x}\right|\right\}$, and its isometric extension

$$
U_{A \rightarrow X E}=\sum_{x}|x\rangle_{X}|x\rangle_{E}\left\langle\left.\phi_{x}\right|_{A} .\right.
$$

For a given state $\rho_{A B}$, the state relevant for discord becomes

$$
\begin{aligned}
U_{A \rightarrow X E} \rho_{A B} U_{A \rightarrow X E}^{\dagger} & =\sum_{x, y}|x\rangle_{X}|x\rangle_{E}\left\langle\left.\phi_{x}\right|_{A} \rho_{A B} \mid \phi_{y}\right\rangle_{A}\left\langley | _ { X } \left\langle\left. y\right|_{E}\right.\right. \\
& =\sum_{x, y}\left\langle\left.\phi_{x}\right|_{A} \rho_{A B} \mid \phi_{y}\right\rangle_{A} \otimes|x\rangle\left\langle\left. y\right|_{X} \otimes \mid x\right\rangle\left\langle\left. y\right|_{E} .\right.
\end{aligned}
$$

The lower bound in (5.43) for this state takes the form:

$$
I(E ; B \mid X) \geq-\log F\left(\rho_{B E X}, \rho_{E X}^{1 / 2} \rho_{X}^{-1 / 2} \rho_{B X} \rho_{X}^{-1 / 2} \rho_{E X}^{1 / 2}\right) .
$$

So, we need to calculate $\rho_{B X}, \rho_{X}$, and $\rho_{E X}$ :

$$
\begin{aligned}
\rho_{B X} & =\sum_{x}\left\langle\left.\phi_{x}\right|_{A} \rho_{A B} \mid \phi_{x}\right\rangle_{A} \otimes|x\rangle\left\langle\left. x\right|_{X},\right. \\
\rho_{X} & =\sum_{x} \operatorname{Tr}\left\{| \phi _ { x } \rangle \langle \phi _ { x } | _ { A } \rho _ { A B } \} | x \rangle \left\langle\left.x\right|_{X},\right.\right. \\
\rho_{E X} & =\sum_{x, y} \operatorname{Tr}\left\{| \phi _ { y } \rangle \langle \phi _ { x } | _ { A } \rho _ { A B } \} | x \rangle \langle y | _ { X } \otimes | x \rangle \left\langle\left.y\right|_{E} .\right.\right.
\end{aligned}
$$

Let

$$
\rho_{B}^{x} \equiv \frac{1}{\operatorname{Tr}\left\{\left|\phi_{x}\right\rangle\left\langle\left.\phi_{x}\right|_{A} \rho_{A B}\right\}\right.}\left\langle\left.\phi_{x}\right|_{A} \rho_{A B} \mid \phi_{x}\right\rangle_{A} .
$$

Then we have that

$$
\begin{aligned}
& \rho_{E X}^{1 / 2} \rho_{X}^{-1 / 2} \rho_{B X} \rho_{X}^{-1 / 2} \rho_{E X}^{1 / 2} \\
& =\rho_{E X}^{1 / 2}\left(\sum_{x}|x\rangle\left\langle\left. x\right|_{X} \otimes \rho_{B}^{x}\right) \rho_{E X}^{1 / 2}\right. \\
& =\left(U_{A \rightarrow X E} \rho_{A} U_{A \rightarrow X E}^{\dagger}\right)^{1 / 2}\left(\sum_{x}|x\rangle\left\langle\left. x\right|_{X} \otimes \rho_{B}^{x}\right)\left(U_{A \rightarrow X E} \rho_{A} U_{A \rightarrow X E}^{\dagger}\right)^{1 / 2}\right. \\
& =U_{A \rightarrow X E} \rho_{A}^{1 / 2} U_{A \rightarrow X E}^{\dagger}\left(\sum_{x}|x\rangle\left\langle\left. x\right|_{X} \otimes \rho_{B}^{x}\right) U_{A \rightarrow X E} \rho_{A}^{1 / 2} U_{A \rightarrow X E}^{\dagger} .\right.
\end{aligned}
$$


Sandwiching by $U_{A \rightarrow X E}^{\dagger}(\cdot) U_{A \rightarrow X E}$ then gives

$$
\begin{aligned}
& \rho_{A}^{1 / 2} U_{A \rightarrow X E}^{\dagger}\left(\sum_{x}|x\rangle\left\langle\left. x\right|_{X} \otimes \rho_{B}^{x}\right) U_{A \rightarrow X E} \rho_{A}^{1 / 2}\right. \\
& =\rho_{A}^{1 / 2}\left(\sum _ { z } | \phi _ { z } \rangle _ { A } \left\langlez | _ { X } \langle z | _ { E } ) \left(\sum _ { x } | x \rangle \langle x | _ { X } \otimes \rho _ { B } ^ { x } ) \left(\sum_{z^{\prime}}\left|z^{\prime}\right\rangle_{X}\left|z^{\prime}\right\rangle_{E}\left\langle\left.\phi_{z^{\prime}}\right|_{A}\right) \rho_{A}^{1 / 2}\right.\right.\right.\right. \\
& =\rho_{A}^{1 / 2}\left(\sum_{z, x, z^{\prime}}\left|\phi_{z}\right\rangle_{A}\left\langle\left.\phi_{z^{\prime}}\right|_{A}\left\langle z|| z^{\prime}\right\rangle_{E}\langle z|| x\rangle\left\langle x|| z^{\prime}\right\rangle_{X} \otimes \rho_{B}^{x}\right) \rho_{A}^{1 / 2}\right. \\
& =\rho_{A}^{1 / 2}\left(\sum_{x}\left|\phi_{x}\right\rangle\left\langle\left.\phi_{x}\right|_{A} \otimes \rho_{B}^{x}\right) \rho_{A}^{1 / 2}\right. \\
& =\sum_{x} \rho_{A}^{1 / 2}\left|\phi_{x}\right\rangle\left\langle\left.\phi_{x}\right|_{A} \rho_{A}^{1 / 2} \otimes \rho_{B}^{x},\right.
\end{aligned}
$$

which we can see is a density operator on systems $A$ and $B$. This establishes that $\rho_{E X}^{1 / 2} \rho_{X}^{-1 / 2} \rho_{B X} \rho_{X}^{-1 / 2} \rho_{E X}^{1 / 2}$ is in the subspace onto which $U_{A \rightarrow X E} U_{A \rightarrow X E}^{\dagger}$ projects, and since this is true also for the state $\rho_{B X E}$, we find that

$$
F\left(\rho_{B E X}, \rho_{E X}^{1 / 2} \rho_{X}^{-1 / 2} \rho_{B X} \rho_{X}^{-1 / 2} \rho_{E X}^{1 / 2}\right)=F\left(\rho_{A B}, \sum_{x} \rho_{A}^{1 / 2}\left|\phi_{x}\right\rangle\left\langle\left.\phi_{x}\right|_{A} \rho_{A}^{1 / 2} \otimes \rho_{B}^{x}\right)\right.
$$

which is the bound in (5.47).

\section{Rényi generalizations of relative entropy differences}

In our prior work, we detailed a procedure to obtain Rényi generalizations of quantum information measures that are equal to linear combinations of von Neumann entropies with coefficients chosen from the set $\{-1,0,1\}$. The procedure relied on writing any given measure as a relative entropy and subsequently using the generalized Lie-Trotter product formula [Suz85] and the Rényi relative entropy [Pet86, $\mathrm{MLDS}^{+} 13$, WWY14] or the sandwiched Rényi relative entropy [MLDS ${ }^{+}$13, WWY14]. In this section, we show that the same procedure can be used to obtain Rényi generalizations of quantum information measures that are equal to differences of relative entropies. Further, we conjecture that the proposed Rényi generalizations of relative entropy differences are monotone in the Rényi parameter. Based on this conjecture, we then suggest remainder terms for two central properties of the relative entropy, namely monotonicity with respect to quantum operations and joint convexity. We also conjecture a remainder term for the Holevo bound.

Consider the following difference of relative entropies:

$$
D(\rho \| \sigma)-D(\mathcal{N}(\rho) \| \mathcal{N}(\sigma)),
$$

where $\rho, \sigma \in \mathcal{S}(\mathcal{H})_{++}$, and $\mathcal{N}$ is a strict CPTP map. The above measure is non-negative, which is a consequence of the monotonicity of relative entropy [Lin74, Lin75]. Writing the adjoint of a CPTP 
map $\mathcal{N}$ as $\mathcal{N}^{\dagger}$, consider that the relative entropy difference in (6.1) can be written as [CL14, ZW14]

$$
\begin{aligned}
& D(\rho \| \sigma)-D(\mathcal{N}(\rho) \| \mathcal{N}(\sigma)) \\
& =\operatorname{Tr}\{\rho[\log \rho-\log \sigma]\}-\operatorname{Tr}\{\mathcal{N}(\rho)[\log \mathcal{N}(\rho)-\log \mathcal{N}(\sigma)]\} \\
& =\operatorname{Tr}\{\rho[\log \rho-\log \sigma]\}-\operatorname{Tr}\left\{\rho\left[\mathcal{N}^{\dagger}(\log \mathcal{N}(\rho))-\mathcal{N}^{\dagger}(\log \mathcal{N}(\sigma))\right]\right\} \\
& =\operatorname{Tr}\{\rho \log \rho\}-\operatorname{Tr}\left\{\rho\left[\log \sigma+\mathcal{N}^{\dagger}(\log \mathcal{N}(\rho)-\log \mathcal{N}(\sigma))\right]\right\} \\
& =D\left(\rho \| \exp \left\{\log \sigma+\mathcal{N}^{\dagger}(\log \mathcal{N}(\rho)-\log \mathcal{N}(\sigma))\right\}\right)
\end{aligned}
$$

In order to find Rényi generalizations of the above quantity, we consider the following lemma:

Lemma 24 Let $\rho, \sigma \in \mathcal{S}(\mathcal{H})_{++}$and $\mathcal{N}$ be a strict CPTP map. Then

$$
\begin{aligned}
\lim _{p \rightarrow 0}\left[\sigma^{p / 2} \mathcal{N}^{\dagger}\left([\mathcal{N}(\sigma)]^{-p / 2}[\mathcal{N}(\rho)]^{p}[\mathcal{N}(\sigma)]^{-p / 2}\right) \sigma^{p / 2}\right]^{1 / p} \\
\quad=\exp \left\{\log \sigma+\mathcal{N}^{\dagger}(\log \mathcal{N}(\rho)-\log \mathcal{N}(\sigma))\right\}
\end{aligned}
$$

Proof. The method of proof is the same as that given for [AH13, Eq. (2.2)]. Consider that

$$
\begin{aligned}
\sigma^{p / 2} \mathcal{N}^{\dagger}\left([\mathcal{N}(\sigma)]^{-p / 2}[\mathcal{N}(\rho)]^{p}[\mathcal{N}(\sigma)]^{-p / 2}\right) \sigma^{p / 2} \\
=\left(I+\frac{p}{2} \log \sigma+o(p)\right) \times \\
\quad \mathcal{N}^{\dagger}\left(\left[I-\frac{p}{2} \log \mathcal{N}(\sigma)+o(p)\right][I+p \log \mathcal{N}(\rho)+o(p)]\left[I-\frac{p}{2} \log \mathcal{N}(\sigma)+o(p)\right]\right) \times \\
\quad\left(I+\frac{p}{2} \log \sigma+o(p)\right) \\
=I+p \log \sigma-p \mathcal{N}^{\dagger}(\log \mathcal{N}(\sigma))+p \mathcal{N}^{\dagger}(\log \mathcal{N}(\rho))+o(p) \\
=I+p\left[\log \sigma+\mathcal{N}^{\dagger}(\log \mathcal{N}(\rho)-\log \mathcal{N}(\sigma))\right]+o(p),
\end{aligned}
$$

where $o(p)$ means that $o(p) / p \rightarrow 0$ in the operator norm as $p \rightarrow 0$. The first equality follows from the Taylor expansion $X^{p}=I+p \log X+o(p)$, and the second equality follows because $\mathcal{N}^{\dagger}(I)=I$. So this implies for $p$ small enough that

$$
\begin{aligned}
& \frac{1}{p} \log \left[\sigma^{p / 2} \mathcal{N}^{\dagger}\left([\mathcal{N}(\sigma)]^{-p / 2}[\mathcal{N}(\rho)]^{p}[\mathcal{N}(\sigma)]^{-p / 2}\right) \sigma^{p / 2}\right] \\
& =\frac{1}{p} \log \left[I+p\left[\log \sigma+\mathcal{N}^{\dagger}(\log \mathcal{N}(\rho)-\log \mathcal{N}(\sigma))\right]+o(p)\right] \\
& =\log \sigma+\mathcal{N}^{\dagger}(\log \mathcal{N}(\rho)-\log \mathcal{N}(\sigma))+o(p)
\end{aligned}
$$

By exponentiating the first and last line and taking the limit as $p \rightarrow 0$, we recover the statement of the lemma.

Appealing to Lemma 24, we can write the second argument of the relative entropy in (6.5) as in (6.6). Based on the procedure given in [BSW15a, Section 10], this suggests the following Rényi generalizations of the relative entropy difference in (6.1) using the Rényi relative entropy and the sandwiched Rényi relative entropy. 
Definition 25 Let $\rho, \sigma \in \mathcal{S}(\mathcal{H})_{++}$and $\mathcal{N}$ be a strict CPTP map. The Rényi generalizations of the relative entropy difference in (6.1) are defined respectively as

$$
\begin{aligned}
& \Delta_{\alpha}(\rho, \sigma, \mathcal{N}) \equiv \frac{1}{\alpha-1} \log \operatorname{Tr}\left\{\rho^{\alpha} \sigma^{(1-\alpha) / 2} \mathcal{N}^{\dagger}\left([\mathcal{N}(\sigma)]^{(\alpha-1) / 2}[\mathcal{N}(\rho)]^{1-\alpha}[\mathcal{N}(\sigma)]^{(\alpha-1) / 2}\right) \sigma^{(1-\alpha) / 2}\right\}, \\
& \widetilde{\Delta}_{\alpha}(\rho, \sigma, \mathcal{N}) \equiv \\
& \frac{\alpha}{\alpha-1} \log \operatorname{Tr}\left\|\rho^{1 / 2} \sigma^{(1-\alpha) / 2 \alpha} \mathcal{N}^{\dagger}\left([\mathcal{N}(\sigma)]^{(\alpha-1) / 2 \alpha}[\mathcal{N}(\rho)]^{(1-\alpha) / \alpha}[\mathcal{N}(\sigma)]^{(\alpha-1) / 2 \alpha}\right) \sigma^{(1-\alpha) / 2 \alpha} \rho^{1 / 2}\right\|_{\alpha} .
\end{aligned}
$$

Both of these quantities converge to (6.1) in the limit as $\alpha \rightarrow 1$, which follows from an argument similar to the proof of [BSW15a, Theorems 9 and 20] (alternatively, one could employ Lemma 24, (6.5), and the fact that the Rényi and sandwiched Rényi relative entropies converge to $D(\rho \| \sigma)$ as $\alpha \rightarrow 1$ and are continuous with respect to the second argument when it is positive definite). We now show that the above Rényi generalizations of a difference of two relative entropies are consistent with the Rényi conditional mutual information proposed earlier in BSW15a, Sections 5 and 6]. Recall that the conditional mutual information can be written as

$$
\begin{aligned}
I(A ; B \mid C) & =I(B ; A C)-I(B ; C) \\
& =D\left(\rho_{A B C} \| \rho_{B} \otimes \rho_{A C}\right)-D\left(\rho_{B C} \| \rho_{B} \otimes \rho_{C}\right),
\end{aligned}
$$

and choosing in (6.12) and (6.13),

$$
\rho=\rho_{A B C}, \quad \sigma=\rho_{B} \otimes \rho_{A C}, \quad \mathcal{N}(\cdot)=\operatorname{Tr}_{A}\{\cdot\},
$$

so that $\mathcal{N}^{\dagger}(\cdot)=(\cdot) \otimes I_{A}$, leads to

$$
\Delta_{\alpha}\left(\rho_{A B C}, \rho_{B} \otimes \rho_{A C}, \operatorname{Tr}_{A}\{\cdot\}\right)=\frac{1}{\alpha-1} \log \operatorname{Tr}\left\{\rho_{A B C}^{\alpha} \rho_{B}^{0} \rho_{A C}^{(1-\alpha) / 2} \rho_{C}^{(\alpha-1) / 2} \rho_{B C}^{1-\alpha} \rho_{C}^{(\alpha-1) / 2} \rho_{A C}^{(1-\alpha) / 2} \rho_{B}^{0}\right\},
$$

as well as

$$
\begin{aligned}
& \widetilde{\Delta}_{\alpha}\left(\rho_{A B C}, \rho_{B} \otimes \rho_{A C}, \operatorname{Tr}_{A}\{\cdot\}\right) \\
& =\frac{\alpha}{\alpha-1} \log \left\|\rho_{A B C}^{1 / 2} \rho_{B}^{0} \rho_{A C}^{(1-\alpha) / 2 \alpha} \rho_{C}^{(\alpha-1) / 2 \alpha} \rho_{B C}^{(1-\alpha) / \alpha} \rho_{C}^{(\alpha-1) / 2 \alpha} \rho_{A C}^{(1-\alpha) / 2 \alpha} \rho_{B}^{0} \rho_{A B C}^{1 / 2}\right\|_{\alpha} .
\end{aligned}
$$

We conclude that the expressions in (6.17) and (6.18) are equal to the Rényi generalizations of the conditional quantum mutual information proposed in BSW15a].

\subsection{Conjectured remainder term for monotonicity of relative entropy}

[BSW15a, Conjecture 34] leads us to one more conjecture, that the above generalizations $\Delta_{\alpha}(\rho, \sigma, \mathcal{N})$ and $\widetilde{\Delta}_{\alpha}(\rho, \sigma, \mathcal{N})$ are monotone in the Rényi parameter as well:

Conjecture 26 Let $\rho, \sigma \in \mathcal{S}(\mathcal{H})_{++}$and $\mathcal{N}$ be a strict CPTP map. Then the following inequalities hold for $0 \leq \alpha \leq \beta$ :

$$
\begin{aligned}
& \Delta_{\alpha}(\rho, \sigma, \mathcal{N}) \leq \Delta_{\beta}(\rho, \sigma, \mathcal{N}) \\
& \widetilde{\Delta}_{\alpha}(\rho, \sigma, \mathcal{N}) \leq \widetilde{\Delta}_{\beta}(\rho, \sigma, \mathcal{N}) .
\end{aligned}
$$


When $\rho, \sigma \in \mathcal{S}(\mathcal{H})_{++}$, and $\mathcal{N}$ is a strict CPTP map, it is possible prove Conjecture 26 in some special cases. For example, when $\alpha$ is in a neighborhood of one, the derivatives of $\Delta_{\alpha}(\rho, \sigma, \mathcal{N})$ and $\widetilde{\Delta}_{\alpha}(\rho, \sigma, \mathcal{N})$ with respect to $\alpha$ are non-negative, as is seen using Taylor expansions of these functions (see Appendix $\mathrm{B}$ for details). This implies that monotonicity holds when $\alpha$ is in a neighborhood of one. Further, when $\alpha$ and $\beta$ satisfy $0 \leq \alpha \leq \beta, \alpha+\beta=2$, and $\mathcal{N}$ is a unitary quantum channel, the inequality in (6.19) for $\Delta_{\alpha}(\rho, \sigma, \mathcal{N})$ is true. This is because, in such a case, we have that $1-\alpha=-(1-\beta)$, so that

$$
\begin{aligned}
{\left[\sigma^{(1-\alpha) / 2} \mathcal{N}^{\dagger}\left([\mathcal{N}(\sigma)]^{(\alpha-1) / 2}[\mathcal{N}(\rho)]^{1-\alpha}[\mathcal{N}(\sigma)]^{(\alpha-1) / 2}\right) \sigma^{(1-\alpha) / 2}\right]^{1 /(1-\alpha)} } \\
\quad=\left[\sigma^{(1-\beta) / 2} \mathcal{N}^{\dagger}\left([\mathcal{N}(\sigma)]^{(\beta-1) / 2}[\mathcal{N}(\rho)]^{1-\beta}[\mathcal{N}(\sigma)]^{(\beta-1) / 2}\right) \sigma^{(1-\beta) / 2}\right]^{1 /(1-\beta)}
\end{aligned}
$$

Then the monotonicity follows from the ordinary monotonicity of the Rényi relative entropy, namely the statement that $D_{\alpha}(\rho \| \sigma) \leq D_{\beta}(\rho \| \sigma)$ for $0 \leq \alpha \leq \beta$ [TCR09]. By a similar line of reasoning, when $1 / \alpha+1 / \beta=2,0 \leq \alpha \leq \beta$, and $\mathcal{N}$ is a unitary quantum channel, the inequality in (6.20) for $\widetilde{\Delta}_{\alpha}(\rho, \sigma, \mathcal{N})$ holds. This is because in such a case, we have that $\alpha /(1-\alpha)=-\beta /(1-\beta)$, so that

$$
\begin{aligned}
& {\left[\sigma^{(1-\alpha) / 2 \alpha} \mathcal{N}^{\dagger}\left([\mathcal{N}(\sigma)]^{(\alpha-1) / 2 \alpha}[\mathcal{N}(\rho)]^{(1-\alpha) / \alpha}[\mathcal{N}(\sigma)]^{(\alpha-1) / 2 \alpha}\right) \sigma^{(1-\alpha) / 2 \alpha}\right]^{\alpha /(1-\alpha)}} \\
& =\left[\sigma^{(1-\beta) / 2 \beta} \mathcal{N}^{\dagger}\left([\mathcal{N}(\sigma)]^{(\beta-1) / 2 \beta}[\mathcal{N}(\rho)]^{(1-\beta) / \beta}[\mathcal{N}(\sigma)]^{(-1) / 2 \beta}\right) \sigma^{(1-\beta) / 2 \beta}\right]^{\beta /(1-\beta)}
\end{aligned}
$$

Since this is the case, the monotonicity follows directly from the ordinary monotonicity of the sandwiched Rényi relative entropy, namely, $\widetilde{D}_{\alpha}(\rho \| \sigma) \leq \widetilde{D}_{\beta}(\rho \| \sigma)$ for $0 \leq \alpha \leq \beta$ [MLDS ${ }^{+} 13$.

We also tested the inequalities in (6.19) and (6.20) numerically for states $\rho$ and $\sigma$ of dimensions up to five, and channels $\mathcal{N}$ of input and output dimensions (not necessarily the same) up to five, chosen at random according to the Haar measure [Cub09]. For $\alpha$ and $\beta$ chosen in the range $(0,1) \cup(1,10]$ such that $\alpha \leq \beta$, we found that the inequalities in (6.19) and (6.20) were never falsified in 1000 numerical runs for each setting of the parameters $\alpha$ and $\beta$.

A consequence of the above conjecture is the following inequality (a similar conjecture can also be found in [Zha14, Eq. (4.7)]). This bound, if proven true, would give an operationally useful answer to a question posed in WL12.

Consequence 27 Let $\rho, \sigma \in \mathcal{S}(\mathcal{H})_{++}$, and $\mathcal{N}$ be a strict CPTP map. Let $\mathcal{T}$ denote the Petz recovery channel, which is indeed a CPTP map for $\rho, \sigma$ and $\mathcal{N}$ as given:

$$
\mathcal{T}(\omega) \equiv \sigma^{1 / 2} \mathcal{N}^{\dagger}\left([\mathcal{N}(\sigma)]^{-1 / 2} \omega[\mathcal{N}(\sigma)]^{-1 / 2}\right) \sigma^{1 / 2}
$$

Assuming Conjecture [26, the relative entropy difference of (6.1) obeys the following lower bounds:

$$
D(\rho \| \sigma)-D(\mathcal{N}(\rho) \| \mathcal{N}(\sigma)) \geq-\log F(\rho, \mathcal{T}(\mathcal{N}(\rho))) .
$$

Proof. Conjecture 26 directly implies the lower bound:

$$
\begin{aligned}
\widetilde{\Delta}_{1}(\rho, \sigma, \mathcal{N}) & \geq \widetilde{\Delta}_{1 / 2}(\rho, \sigma, \mathcal{N}) \\
\Leftrightarrow D(\rho \| \sigma)-D(\mathcal{N}(\rho) \| \mathcal{N}(\sigma)) & \geq-\log \operatorname{Tr}\left\{\sqrt{\rho^{1 / 2} \mathcal{T}(\mathcal{N}(\rho)) \rho^{1 / 2}}\right\} \\
& =-\log F(\rho, \mathcal{T}(\mathcal{N}(\rho))) .
\end{aligned}
$$




\subsection{Conjectured remainder term for joint convexity of relative entropy}

We now use (6.24) to suggest a remainder term for joint convexity of relative entropy. We once again emphasize that this remainder term is a consequence of Conjecture 26. Interestingly, this inequality is equivalent to the inequality in (6.24).

Consequence 28 Let $p_{X}(x)$ be a probability distribution characterizing the ensembles $\left\{p_{X}(x), \rho_{x}\right\}$ and $\left\{p_{X}(x), \sigma_{x}\right\}$ with $\rho_{x}, \sigma_{x} \in \mathcal{S}(\mathcal{H})_{++}$. From Consequence 27 we have the following remainder term for joint convexity of relative entropy:

$$
\sum_{x} p_{X}(x) D\left(\rho_{x} \| \sigma_{x}\right)-D(\bar{\rho} \| \bar{\sigma}) \geq-2 \log \left(\sum_{x} p_{X}(x) \sqrt{F\left(\rho_{x}, \sigma_{x}^{1 / 2}(\bar{\sigma})^{-1 / 2} \bar{\rho}(\bar{\sigma})^{-1 / 2} \sigma_{x}^{1 / 2}\right)}\right),
$$

where $\bar{\rho}=\sum_{x} p_{X}(x) \rho_{x}$ and $\bar{\sigma}=\sum_{x} p_{X}(x) \sigma_{x}$. Furthermore, the inequality (6.28) implies and is implied by the inequality (6.24) (Consequence 27), so that these inequalities are equivalent.

Proof. Let

$$
\begin{aligned}
\rho_{X B} & \equiv \sum_{x} p_{X}(x)|x\rangle\langle x| \otimes \rho_{x}, \\
\sigma_{X B} & \equiv \sum_{x} p_{X}(x)|x\rangle\langle x| \otimes \sigma_{x}, \\
\bar{\rho} & \equiv \rho_{B}=\operatorname{Tr}_{X}\left\{\rho_{X B}\right\}=\sum_{x} p_{X}(x) \rho_{x} \\
\bar{\sigma} & \equiv \sigma_{B}=\operatorname{Tr}_{X}\left\{\sigma_{X B}\right\}=\sum_{x} p_{X}(x) \sigma_{x}
\end{aligned}
$$

Consider that (6.24) with the map $\mathcal{N}$ being a trace-out map over system $X$ implies that

$$
\begin{aligned}
\sum_{x} p(x) D\left(\rho_{x} \| \sigma_{x}\right)-D(\bar{\rho} \| \bar{\sigma}) & =D\left(\rho_{X B} \| \sigma_{X B}\right)-D\left(\rho_{B} \| \sigma_{B}\right) \\
& \geq-\log F\left(\rho_{X B}, \sigma_{X B}^{1 / 2} \sigma_{B}^{-1 / 2} \rho_{B} \sigma_{B}^{-1 / 2} \sigma_{X B}^{1 / 2}\right) \\
& =-2 \log \left(\sum_{x} p(x) \sqrt{F\left(\rho_{x}, \sigma_{x}^{1 / 2}(\bar{\sigma})^{-1 / 2} \bar{\rho}(\bar{\sigma})^{-1 / 2} \sigma_{x}^{1 / 2}\right)}\right) .
\end{aligned}
$$

This establishes the first claim. Now suppose that

$$
\sum_{x} p(x) D\left(\rho_{x} \| \sigma_{x}\right)-D(\bar{\rho} \| \bar{\sigma}) \geq-2 \log \left(\sum_{x} p(x) \sqrt{F\left(\rho_{x}, \sigma_{x}^{1 / 2}(\bar{\sigma})^{-1 / 2} \bar{\rho}(\bar{\sigma})^{-1 / 2} \sigma_{x}^{1 / 2}\right)}\right)
$$

is true. We pick

$$
\begin{aligned}
\rho_{x} & =V_{E}^{i} U_{A E}\left(\omega_{A} \otimes|0\rangle\left\langle\left. 0\right|_{E}\right) U_{A E}^{\dagger}\left(V_{E}^{i}\right)^{\dagger},\right. \\
\sigma_{x} & =V_{E}^{i} U_{A E}\left(\tau_{A} \otimes|0\rangle\left\langle\left. 0\right|_{E}\right) U_{A E}^{\dagger}\left(V_{E}^{i}\right)^{\dagger},\right. \\
p(x) & =\frac{1}{d_{E}^{2}}
\end{aligned}
$$


with $\left\{V_{E}^{i}\right\}_{i=1}^{d_{E}^{2}}$ a Heisenberg-Weyl set of unitaries. These choices imply that

$$
\bar{\rho}=\mathcal{N}\left(\omega_{A}\right) \otimes \pi_{E}, \quad \bar{\sigma}=\mathcal{N}\left(\tau_{A}\right) \otimes \pi_{E},
$$

where $\pi_{E}$ is the maximally mixed state on system $E$, and $\mathcal{N}$ is defined by

$$
\mathcal{N}(\cdot)=\operatorname{Tr}_{E}\left\{U_{A E}\left[(\cdot) \otimes|0\rangle\left\langle\left. 0\right|_{E}\right] U_{A E}^{\dagger}\right\}=\sum_{j}\left\langle\left. j\right|_{E} U_{A E} \mid 0\right\rangle_{E}(\cdot)\left\langle\left. 0\right|_{E} U_{A E}^{\dagger} \mid j\right\rangle_{E},\right.
$$

so that the Kraus operators of $\mathcal{N}$ are $\left\{\left\langle\left. j\right|_{E} U_{A E} \mid 0\right\rangle_{E}\right\}_{j}$. Then the adjoint map $\mathcal{N}^{\dagger}$ is given by

$$
\mathcal{N}^{\dagger}(\cdot)=\sum_{j}\left\langle\left. 0\right|_{E} U_{A E}^{\dagger} \mid j\right\rangle_{E}(\cdot)\left\langle\left. j\right|_{E} U_{A E} \mid 0\right\rangle_{E} \cdot
$$

Furthermore, we have that

$$
\begin{aligned}
\sum_{x} p(x) D\left(\rho_{x} \| \sigma_{x}\right) & =D\left(\omega_{A} \| \tau_{A}\right) \\
D(\bar{\rho} \| \bar{\sigma}) & =D\left(\mathcal{N}\left(\omega_{A}\right) \| \mathcal{N}\left(\tau_{A}\right)\right)
\end{aligned}
$$

Consider that

$$
\begin{gathered}
\sigma_{x}^{1 / 2}(\bar{\sigma})^{-1 / 2} \bar{\rho}(\bar{\sigma})^{-1 / 2} \sigma_{x}^{1 / 2} \\
=\left[V_{E}^{i} U_{A E}\left(\tau_{A} \otimes|0\rangle\left\langle\left. 0\right|_{E}\right) U_{A E}^{\dagger}\left(V_{E}^{i}\right)^{\dagger}\right]^{1 / 2}\left[\mathcal{N}\left(\tau_{A}\right) \otimes \pi_{E}\right]^{-1 / 2}\left[\mathcal{N}\left(\omega_{A}\right) \otimes \pi_{E}\right] \times\right. \\
{\left[\mathcal{N}\left(\tau_{A}\right) \otimes \pi_{E}\right]^{-1 / 2}\left[V_{E}^{i} U_{A E}\left(\tau_{A} \otimes|0\rangle\left\langle\left. 0\right|_{E}\right) U_{A E}^{\dagger}\left(V_{E}^{i}\right)^{\dagger}\right]^{1 / 2}\right.} \\
=V_{E}^{i} U_{A E}\left(\tau_{A}^{1 / 2} \otimes|0\rangle\left\langle\left. 0\right|_{E}\right) U_{A E}^{\dagger}\left(V_{E}^{i}\right)^{\dagger}\left(\left[\mathcal{N}\left(\tau_{A}\right)\right]^{-1 / 2} \mathcal{N}\left(\omega_{A}\right)\left[\mathcal{N}\left(\tau_{A}\right)\right]^{-1 / 2} \otimes I_{E}\right) \times\right. \\
V_{E}^{i} U_{A E}\left(\tau_{A}^{1 / 2} \otimes|0\rangle\left\langle\left. 0\right|_{E}\right) U_{A E}^{\dagger}\left(V_{E}^{i}\right)^{\dagger}\right. \\
=V_{E}^{i} U_{A E}\left(\tau_{A}^{1 / 2} \otimes|0\rangle\left\langle\left. 0\right|_{E}\right) U_{A E}^{\dagger}\left(\left[\mathcal{N}\left(\tau_{A}\right)\right]^{-1 / 2} \mathcal{N}\left(\omega_{A}\right)\left[\mathcal{N}\left(\tau_{A}\right)\right]^{-1 / 2} \otimes I_{E}\right) \times\right. \\
U_{A E}\left(\tau_{A}^{1 / 2} \otimes|0\rangle\left\langle\left. 0\right|_{E}\right) U_{A E}^{\dagger}\left(V_{E}^{i}\right)^{\dagger}\right. \\
=V_{E}^{i} U_{A E}\left(\tau _ { A } ^ { 1 / 2 } \otimes | 0 \rangle \langle 0 | _ { E } ) U _ { A E } ^ { \dagger } \left(\left[\mathcal{N}\left(\tau_{A}\right)\right]^{-1 / 2} \mathcal{N}\left(\omega_{A}\right)\left[\mathcal{N}\left(\tau_{A}\right)\right]^{-1 / 2} \otimes \sum_{j}|j\rangle\left\langle\left. j\right|_{E}\right) \times\right.\right. \\
U_{A E}\left(\tau_{A}^{1 / 2} \otimes|0\rangle\left\langle\left. 0\right|_{E}\right) U_{A E}^{\dagger}\left(V_{E}^{i}\right)^{\dagger}\right. \\
=V_{E}^{i} U_{A E}\left(\tau_{A}^{1 / 2} \otimes|0\rangle_{E}\right) \sum_{j}\left\langle\left. 0\right|_{E} U_{A E}^{\dagger} \mid j\right\rangle_{E}\left(\left[\mathcal{N}\left(\tau_{A}\right)\right]^{-1 / 2} \mathcal{N}\left(\omega_{A}\right)\left[\mathcal{N}\left(\tau_{A}\right)\right]^{-1 / 2}\right)\left\langle\left. j\right|_{E} U_{A E} \mid 0\right\rangle_{E} \times \\
\left(\tau_{A}^{1 / 2} \otimes\left\langle\left. 0\right|_{E}\right) U_{A E}^{\dagger}\left(V_{E}^{i}\right)^{\dagger}\right. \\
=V_{E}^{i} U_{A E}\left(\tau_{A}^{1 / 2} \otimes|0\rangle_{E}\right) \mathcal{N}^{\dagger}\left(\left[\mathcal{N}\left(\tau_{A}\right)\right]^{-1 / 2} \mathcal{N}\left(\omega_{A}\right)\left[\mathcal{N}\left(\tau_{A}\right)\right]^{-1 / 2}\right)\left(\tau_{A}^{1 / 2} \otimes\left\langle\left. 0\right|_{E}\right) U_{A E}^{\dagger}\left(V_{E}^{i}\right)^{\dagger}\right. \\
=V_{E}^{i} U_{A E}\left(\left[\tau_{A}^{1 / 2} \mathcal{N} \dagger\left(\left[\mathcal{N}\left(\tau_{A}\right)\right]^{-1 / 2} \mathcal{N}\left(\omega_{A}\right)\left[\mathcal{N}\left(\tau_{A}\right)\right]^{-1 / 2}\right) \tau_{A}^{1 / 2}\right] \otimes|0\rangle\left\langle\left. 0\right|_{E}\right) U_{A E}^{\dagger}\left(V_{E}^{i}\right)^{\dagger}\right.
\end{gathered}
$$


So two to the power of minus one half times the remainder term of joint convexity evaluates to

$$
\begin{aligned}
& \sum_{x} p(x) \sqrt{F\left(\rho_{x}, \sigma_{x}^{1 / 2}(\bar{\sigma})^{-1 / 2} \bar{\rho}(\bar{\sigma})^{-1 / 2} \sigma_{x}^{1 / 2}\right)} \\
& =\sum_{i} \frac{1}{d_{E}^{2}} F^{1 / 2}\left(V _ { E } ^ { i } U _ { A E } \left(\omega_{A} \otimes|0\rangle\left\langle\left. 0\right|_{E}\right) U_{A E}^{\dagger}\left(V_{E}^{i}\right)^{\dagger},\right.\right. \\
& V_{E}^{i} U_{A E}\left(\left[\tau_{A}^{1 / 2} \mathcal{N}^{\dagger}\left(\left[\mathcal{N}\left(\tau_{A}\right)\right]^{-1 / 2} \mathcal{N}\left(\omega_{A}\right)\left[\mathcal{N}\left(\tau_{A}\right)\right]^{-1 / 2}\right) \tau_{A}^{1 / 2}\right] \otimes|0\rangle\left\langle\left. 0\right|_{E}\right) U_{A E}^{\dagger}\left(V_{E}^{i}\right)^{\dagger}\right) \\
& =\sum_{i} \frac{1}{d_{E}^{2}} \sqrt{F\left(\omega_{A} \otimes|0\rangle\left\langle\left. 0\right|_{E},\left[\tau_{A}^{1 / 2} \mathcal{N}^{\dagger}\left(\left[\mathcal{N}\left(\tau_{A}\right)\right]^{-1 / 2} \mathcal{N}\left(\omega_{A}\right)\left[\mathcal{N}\left(\tau_{A}\right)\right]^{-1 / 2}\right) \tau_{A}^{1 / 2}\right] \otimes \mid 0\right\rangle\left\langle\left. 0\right|_{E}\right)\right.} \\
& =\sqrt{F\left(\omega_{A}, \tau_{A}^{1 / 2} \mathcal{N}^{\dagger}\left(\left[\mathcal{N}\left(\tau_{A}\right)\right]^{-1 / 2} \mathcal{N}\left(\omega_{A}\right)\left[\mathcal{N}\left(\tau_{A}\right)\right]^{-1 / 2}\right) \tau_{A}^{1 / 2}\right)}
\end{aligned}
$$

which gives (6.24).

\subsection{A conjectured remainder term for the Holevo bound}

The Holevo bound [Hol73] is the statement that the following inequality holds

$$
I(X ; B)_{\rho} \geq I(X ; Y)_{\omega},
$$

where

$$
\rho_{X B} \equiv \sum_{x} p_{X}(x)|x\rangle\left\langle\left. x\right|_{X} \otimes \rho_{B}^{x}, \quad \omega_{X Y}=\mathcal{M}_{B \rightarrow Y}\left(\rho_{X B}\right),\right.
$$

and $\mathcal{M}_{B \rightarrow Y}$ is a measurement channel defined as

$$
\mathcal{M}_{B \rightarrow Y}\left(\sigma_{B}\right) \equiv \sum_{y}\left\langle\varphi^{y}\left|\sigma_{B}\right| \varphi^{y}\right\rangle|y\rangle\left\langle\left. y\right|_{Y}, \quad \sum_{y} \mid \varphi^{y}\right\rangle\left\langle\left.\varphi^{y}\right|_{B}=I_{B}\right.
$$

The Kraus operators for the measurement channel $\mathcal{M}_{B \rightarrow Y}$ are $\left\{|y\rangle\left\langle\varphi^{y}\right|\right\}$. (One might as well restrict to rank-one measurements since these already give a tighter lower bound in (6.55) than arbitrary measurements.) We now write down a lower bound on the Holevo bound based on Consequence 27. The interpretation of the lower bound is that it quantifies how far away the states $\rho^{x}$ are from commuting. If the difference between the Holevo information and the classical mutual information is equal to zero, then the bound below holds with equality. In this case, each state $\rho^{x}$ is a fixed point of the same entanglement-breaking channel, which in turn by [FNW15, Theorem 5.3] implies that the states are commuting (a result well known since Holevo's original work).

Consequence 29 The following remainder term for the Holevo bound is a consequence of the conjecture in (6.24):

$$
I(X ; B)_{\rho}-I(X ; Y)_{\omega} \geq-2 \log \left(\sum_{x} p_{X}(x) \sqrt{F\left(\rho_{B}^{x}, \mathcal{E}_{B}\left(\rho_{B}^{x}\right)\right)}\right),
$$

where $\mathcal{E}_{B}$ is an entanglement-breaking channel defined as

$$
\mathcal{E}_{B}\left(\sigma_{B}\right)=\sum_{y}\left\langle\varphi^{y}\left|\sigma_{B}\right| \varphi^{y}\right\rangle \frac{\rho_{B}^{1 / 2}\left|\varphi^{y}\right\rangle\left\langle\left.\varphi^{y}\right|_{B} \rho_{B}^{1 / 2}\right.}{\left\langle\varphi^{y}\left|\rho_{B}\right| \varphi^{y}\right\rangle} .
$$


Proof. We would like to determine remainder terms for the inequality in (6.55) and can do so by rewriting it in terms of relative entropies:

$$
D\left(\rho_{X B} \| \rho_{X} \otimes \rho_{B}\right)-D\left(\omega_{X Y} \| \omega_{X} \otimes \omega_{Y}\right) \geq 0 .
$$

We now apply the conjecture in (6.24) to the case of the Holevo bound with

$$
\rho=\rho_{X B}, \quad \sigma=\rho_{X} \otimes \rho_{B}, \quad \mathcal{N}=\operatorname{id}_{X} \otimes \mathcal{M}_{B \rightarrow Y} .
$$

Consider for these choices that

$$
\begin{aligned}
\mathcal{N}(\rho) & =\omega_{X Y}=\sum_{x, y} p_{X}(x)\left\langle\varphi^{y}\left|\rho_{B}^{x}\right| \varphi^{y}\right\rangle|x\rangle\left\langle\left. x\right|_{X} \otimes \mid y\right\rangle\left\langle\left. y\right|_{Y},\right. \\
{[\mathcal{N}(\sigma)]^{-1 / 2} } & =\left[\rho_{X} \otimes \mathcal{M}_{B \rightarrow Y}\left(\rho_{B}\right)\right]^{-1 / 2} \\
& =\rho_{X}^{-1 / 2} \otimes\left[\mathcal{M}_{B \rightarrow Y}\left(\rho_{B}\right)\right]^{-1 / 2} \\
& =\sum_{x}\left[p_{X}(x)\right]^{-1 / 2}|x\rangle\left\langle\left. x\right|_{X} \otimes \sum_{y}\left[\left\langle\varphi^{y}\left|\rho_{B}\right| \varphi^{y}\right\rangle\right]^{-1 / 2} \mid y\right\rangle\left\langle\left. y\right|_{Y},\right. \\
\mathcal{M}_{B \rightarrow Y}^{\dagger}(\cdot) & =\sum_{y}\left|\varphi^{y}\right\rangle\langle y|(\cdot)| y\rangle\left\langle\varphi^{y}\right| \\
\sigma^{1 / 2} & =\rho_{X}^{1 / 2} \otimes \rho_{B}^{1 / 2} \\
& =\sum_{x}\left[p_{X}(x)\right]^{1 / 2}|x\rangle\left\langle\left. x\right|_{X} \otimes \rho_{B}^{1 / 2} .\right.
\end{aligned}
$$

So we find that

$$
[\mathcal{N}(\sigma)]^{-1 / 2} \mathcal{N}(\rho)[\mathcal{N}(\sigma)]^{-1 / 2}=\sum_{x, y}|x\rangle\left\langle\left. x\right|_{X} \otimes \frac{\left\langle\varphi^{y}\left|\rho_{B}^{x}\right| \varphi^{y}\right\rangle}{\left\langle\varphi^{y}\left|\rho_{B}\right| \varphi^{y}\right\rangle} \mid y\right\rangle\left\langle\left. y\right|_{Y}\right.
$$

and then

$$
\begin{aligned}
\mathcal{N}^{\dagger}\left([\mathcal{N}(\sigma)]^{-1 / 2} \mathcal{N}(\rho)[\mathcal{N}(\sigma)]^{-1 / 2}\right) & =\mathcal{M}_{B \rightarrow Y}^{\dagger}\left(\sum_{x, y}|x\rangle\left\langle\left. x\right|_{X} \otimes \frac{\left\langle\varphi^{y}\left|\rho_{B}^{x}\right| \varphi^{y}\right\rangle}{\left\langle\varphi^{y}\left|\rho_{B}\right| \varphi^{y}\right\rangle} \mid y\right\rangle\left\langle\left. y\right|_{Y}\right)\right. \\
& =\sum_{x, y}|x\rangle\left\langle\left. x\right|_{X} \otimes \frac{\left\langle\varphi^{y}\left|\rho_{B}^{x}\right| \varphi^{y}\right\rangle}{\left\langle\varphi^{y}\left|\rho_{B}\right| \varphi^{y}\right\rangle} \mid \varphi^{y}\right\rangle\left\langle\left.\varphi^{y}\right|_{Y} .\right.
\end{aligned}
$$

Finally, we have that

$$
\begin{aligned}
& \sigma^{1 / 2} \mathcal{N}^{\dagger}\left([\mathcal{N}(\sigma)]^{-1 / 2} \mathcal{N}(\rho)[\mathcal{N}(\sigma)]^{-1 / 2}\right) \sigma^{1 / 2} \\
& =\sum_{x, y} p_{X}(x)|x\rangle\left\langle\left. x\right|_{X} \otimes \frac{\left\langle\varphi^{y}\left|\rho_{B}^{x}\right| \varphi^{y}\right\rangle}{\left\langle\varphi^{y}\left|\rho_{B}\right| \varphi^{y}\right\rangle} \rho_{B}^{1 / 2} \mid \varphi^{y}\right\rangle\left\langle\left.\varphi^{y}\right|_{B} \rho_{B}^{1 / 2}\right. \\
& =\sum_{x, y} p_{X}(x) \frac{\left\langle\varphi^{y}\left|\rho_{B}^{x}\right| \varphi^{y}\right\rangle}{\left\langle\varphi^{y}\left|\rho_{B}\right| \varphi^{y}\right\rangle}|x\rangle\left\langle\left. x\right|_{X} \otimes \rho_{B}^{1 / 2} \mid \varphi^{y}\right\rangle\left\langle\left.\varphi^{y}\right|_{B} \rho_{B}^{1 / 2}\right.
\end{aligned}
$$

Defining

$$
\sigma_{X B} \equiv \sum_{x} p_{X}(x)|x\rangle\left\langle\left. x\right|_{X} \otimes \sigma_{B}^{x}\right.
$$


where

$$
\sigma_{B}^{x}=\sum_{y} \frac{\left\langle\varphi^{y}\left|\rho_{B}^{x}\right| \varphi^{y}\right\rangle}{\left\langle\varphi^{y}\left|\rho_{B}\right| \varphi^{y}\right\rangle} \rho_{B}^{1 / 2}\left|\varphi^{y}\right\rangle\left\langle\left.\varphi^{y}\right|_{B} \rho_{B}^{1 / 2}\right.
$$

we see that

$$
\sigma^{1 / 2} \mathcal{N}^{\dagger}\left([\mathcal{N}(\sigma)]^{-1 / 2} \mathcal{N}(\rho)[\mathcal{N}(\sigma)]^{-1 / 2}\right) \sigma^{1 / 2}=\sigma_{X B}
$$

So a conjectured remainder term for the Holevo bound is

$$
\begin{aligned}
I(X ; B)_{\rho}-I(X ; Y)_{\omega} \geq-\log F\left(\rho_{X B}, \sigma_{X B}\right) & =-2 \log \sqrt{F\left(\rho_{X B}, \sigma_{X B}\right)} \\
& =-2 \log \left(\sum_{x} p_{X}(x) \sqrt{F\left(\rho_{B}^{x}, \sigma_{B}^{x}\right)}\right) .
\end{aligned}
$$

\section{Discussion}

This paper has defined and examined various properties of a Rényi squashed entanglement and a Rényi quantum discord. We took as a conjecture that the Rényi conditional mutual information of a tripartite state $\rho_{A B C}$ is monotone with respect to local CPTP maps on both systems $A$ and $B$. Assuming the conjecture, we showed that these quantities retain most of the properties of the original von Neumann entropy-based quantities. For example, we showed that the Rényi squashed entanglement is convex, monotone with respect to LOCC, that it vanishes on separable states and is subadditive on tensor-product states. Similarly, we showed that the Rényi quantum discord is non-negative, invariant with respect to the action of local unitaries, vanishes on the set of classicalquantum states, and is optimized by a rank-one POVM. Further, we proved relations of the Rényi squashed entanglement to a Rényi entropy of entanglement and a Rényi entanglement of formation. We gave an expression for the Rényi discord of pure bipartite states. Also, assuming the truth of a conjecture on the monotonicity of the Rényi conditional quantum mutual information with respect to the Rényi parameter, we derived a remainder term for von Neumann entropy based quantum discord via the Rényi quantum discord.

This paper has also extended the procedure from our prior work for producing Rényi generalizations of quantum information measures to include measures that are equal to differences of relative entropies. By conjecturing that the proposed Rényi generalizations of differences of relative entropies are monotone in the Rényi parameter, we suggested remainder terms for the monotonicity and joint convexity of relative entropy, and the Holevo bound. These remainder terms, if proven true, hold important applications in quantum information theory [WL12, Kim13, Tou14].

The following are some future directions that could be considered based on the results presented in this work. First of all, it remains a pressing open question if the Rényi conditional mutual information is monotone with respect to local operations on both systems $A$ and $B$ (Conjecture 1). If Conjecture 1 is false, then some of the consequences in this paper are directly impacted, such as the LOCC monotonicity of the Rényi squashed entanglement. One could also try to prove more properties of the Rényi squashed entanglement and discord. For example, we have left open the converse part of faithfulness for both the Rényi squashed entanglement as well as the Rényi discord. The von Neumann entropy based squashed entanglement is known to be superadditive in general and additive on tensor-product states. However, we have only been able to show that the 
Rényi squashed entanglement is subadditive on tensor-product states; super-additivity of the Rényi squashed entanglement in general has been left open. As far as applications are concerned, it is an open question if the von Neumann entropy based squashed entanglement is a strong converse rate for entanglement distillation; the Rényi squashed entanglement may be a useful tool in investigating this question. Also, using the Rényi squashed entanglement, one could try to prove that the von Neumann entropy based squashed entanglement is a strong converse rate for the two-way assisted quantum capacity of any channel (the weak converse bound being shown in [TGW14b, TGW14a]). It might also be interesting to determine if a Koashi-Winter type KW04 relation holds for the proposed Rényi discord.

Note: After the completion of this work, we learned of the recent result of [FR14], in which the following inequality was established for any tripartite state $\rho_{A B C} \in \mathcal{S}\left(\mathcal{H}_{A} \otimes \mathcal{H}_{B} \otimes \mathcal{H}_{C}\right)$ :

$$
I(A ; B \mid C)_{\rho} \geq-\log F(A ; B \mid C)_{\rho},
$$

where $F(A ; B \mid C)_{\rho}$ is the fidelity of recovery, defined independently in [SW14] as

$$
F(A ; B \mid C)_{\rho} \equiv \sup _{\mathcal{R}} F\left(\rho_{A B C}, \mathcal{R}_{C \rightarrow A C}\left(\rho_{B C}\right)\right)
$$

The fidelity of recovery quantifies how well one can recover the full state on systems $A B C$ if system $A$ is lost and one is allowed to perform a recovery map on system $C$ alone. Thus, for states with small conditional mutual information (near to zero), the fidelity of recovery is high (near to one). Inequality (7.1) clearly impacts our Consequences 23 and 29, so that one can interpret a state with small quantum discord as being an approximate fixed point of an entanglement breaking channel and an ensemble for which the Holevo information is close to the accessible information as having all states in the ensemble as approximate fixed points of the same entanglement breaking channel.

Acknowledgments. We thank A. R. P. Rau for many helpful discussions about this work and John Calsamiglia for discussions about Rényi quantum discord. KS and MMW are grateful to Naresh Sharma for hosting them for a research visit to the Tata Institute of Fundamental Research during June 2014, where some of the results in this paper were established. MMW is grateful to the Institute for Quantum Information and Matter at Caltech for hospitality during a research visit in July 2014. KS acknowledges support from NSF Grant No. CCF-1350397, the DARPA Quiness Program through US Army Research Office award W31P4Q-12-1-0019, and the Graduate School of Louisiana State University for the 2014-2015 Dissertation Year Fellowship. MMW acknowledges support from the APS-IUSSTF Professorship Awards in Physics, startup funds from the Department of Physics and Astronomy at LSU, support from the NSF under Award No. CCF-1350397, and support from the DARPA Quiness Program through US Army Research Office award W31P4Q-12-1-0019.

\section{A Rényi conditional quantum mutual information: useful lemmas}

\section{A.1 Invariance with respect to tensoring with product states}

Lemma 30 Let $\rho_{A A_{1} B B_{1} E E_{1}} \equiv \omega_{A B E} \otimes \sigma_{A_{1}} \otimes \tau_{B_{1}} \otimes \gamma_{E_{1}}$. Then

$$
I_{\alpha}\left(A A_{1} ; B B_{1} \mid E E_{1}\right)_{\rho}=I_{\alpha}(A ; B \mid E)_{\omega} .
$$


Proof. This follows from a direct calculation. Consider that

$$
\begin{aligned}
& \operatorname{Tr}_{A A_{1}}\left\{\rho_{A A_{1} E E_{1}}^{(1-\alpha) / 2} \rho_{A A_{1} B B_{1} E E_{1}}^{\alpha} \rho_{A A_{1} E E_{1}}^{(1-\alpha) / 2}\right\} \\
& =\operatorname{Tr}_{A A_{1}}\left\{\left(\omega_{A E} \otimes \sigma_{A_{1}} \otimes \gamma_{E_{1}}\right)^{(1-\alpha) / 2}\left(\omega_{A B E} \otimes \sigma_{A_{1}} \otimes \tau_{B_{1}} \otimes \gamma_{E_{1}}\right)^{\alpha}\left(\omega_{A E} \otimes \sigma_{A_{1}} \otimes \gamma_{E_{1}}\right)^{(1-\alpha) / 2}\right\} \\
& =\operatorname{Tr}_{A A_{1}}\left\{\left(\omega_{A E}^{(1-\alpha) / 2} \otimes \sigma_{A_{1}}^{(1-\alpha) / 2} \otimes \gamma_{E_{1}}^{(1-\alpha) / 2}\right)\left(\omega_{A B E}^{\alpha} \otimes \sigma_{A_{1}}^{\alpha} \otimes \tau_{B_{1}}^{\alpha} \otimes \gamma_{E_{1}}^{\alpha}\right)\left(\omega_{A E}^{(1-\alpha) / 2} \otimes \sigma_{A_{1}}^{(1-\alpha) / 2} \otimes \gamma_{E_{1}}^{(1-\alpha) / 2}\right)\right\} \\
& =\operatorname{Tr}_{A A_{1}}\left\{\omega_{A E}^{(1-\alpha) / 2}\left(\omega_{A B E}^{\alpha} \otimes \tau_{B_{1}}^{\alpha}\right) \omega_{A E}^{(1-\alpha) / 2} \otimes \sigma_{A_{1}} \otimes \gamma_{E_{1}}\right\} \\
& =\operatorname{Tr}_{A}\left\{\omega_{A E}^{(1-\alpha) / 2} \omega_{A B E}^{\alpha} \omega_{A E}^{(1-\alpha) / 2}\right\} \otimes \tau_{B_{1}}^{\alpha} \otimes \gamma_{E_{1}} .
\end{aligned}
$$

From the fact that

$$
\begin{aligned}
& \operatorname{Tr}\left\{\left(\rho_{E E_{1}}^{(\alpha-1) / 2} \operatorname{Tr}_{A A_{1}}\left\{\rho_{A A_{1} E E_{1}}^{(1-\alpha) / 2} \rho_{A A_{1} B B_{1} E E_{1}}^{\alpha} \rho_{A A_{1} E E_{1}}^{(1-\alpha) / 2}\right\} \rho_{E E_{1}}^{(\alpha-1) / 2}\right)^{1 / \alpha}\right\} \\
& =\operatorname{Tr}\left\{\left(\left(\omega_{E}^{(\alpha-1) / 2} \otimes \gamma_{E_{1}}^{(\alpha-1) / 2}\right)\left(\operatorname{Tr}_{A}\left\{\omega_{A E E_{1}}^{(1-\alpha) / 2} \omega_{A B E E_{1}}^{\alpha} \omega_{A E E_{1}}^{(1-\alpha) / 2}\right\} \otimes \gamma_{E_{1}}\right)\left(\omega_{E}^{(\alpha-1) / 2} \otimes \gamma_{E_{1}}^{(\alpha-1) / 2}\right) \otimes \tau_{B_{1}}^{\alpha}\right)^{1 / \alpha}\right\} \\
& =\operatorname{Tr}\left\{\left(\omega_{E}^{(\alpha-1) / 2}\left(\operatorname{Tr}_{A}\left\{\omega_{A E E_{1}}^{(1-\alpha) / 2} \omega_{A B E E_{1}}^{\alpha} \omega_{A E E_{1}}^{(1-\alpha) / 2}\right\}\right) \omega_{E}^{(\alpha-1) / 2} \otimes \tau_{B_{1}}^{\alpha} \otimes \gamma_{E_{1}}^{\alpha}\right)^{1 / \alpha}\right\} \\
& =\operatorname{Tr}\left\{\left(\omega_{E}^{(\alpha-1) / 2} \operatorname{Tr}_{A}\left\{\omega_{A E}^{(1-\alpha) / 2} \omega_{A B E^{\alpha}}^{\alpha} \omega_{A E}^{(1-\alpha) / 2}\right\} \omega_{E}^{(\alpha-1) / 2}\right)^{1 / \alpha} \otimes \tau_{B_{1}} \otimes \gamma_{E_{1}}\right\} \\
& =\operatorname{Tr}\left\{\left(\omega_{E}^{(\alpha-1) / 2} \operatorname{Tr}_{A}\left\{\omega_{A E}^{(1-\alpha) / 2} \omega_{A B E^{\alpha}}^{\alpha} \omega_{A E}^{(1-\alpha) / 2}\right\} \omega_{E}^{(\alpha-1) / 2}\right)^{1 / \alpha}\right\}
\end{aligned}
$$

it follows that

$$
I_{\alpha}\left(A A_{1} ; B B_{1} \mid E E_{1}\right)_{\rho}=I_{\alpha}(A ; B \mid E)_{\omega}
$$

\section{A.2 Conditioning on classical information}

Lemma 31 Let $\rho_{X A B C}$ be a classical-quantum state of the following form:

$$
\rho_{X A B C}=\sum_{x} p_{X}(x)|x\rangle\left\langle\left. x\right|_{X} \otimes \rho_{A B C}^{x} .\right.
$$

Then the following identity holds for $\alpha>0$ :

$$
I_{\alpha}(A ; B \mid C X)_{\rho}=\frac{\alpha}{\alpha-1} \log \sum_{x} p_{X}(x) \exp \left\{\left(\frac{\alpha-1}{\alpha}\right) I_{\alpha}(A ; B \mid C)_{\rho^{x}}\right\} .
$$

Proof. Recalling the formula in (3.18), we have

$$
I_{\alpha}(A ; B \mid C X)_{\rho}=\frac{\alpha}{\alpha-1} \log \operatorname{Tr}\left\{\left(\operatorname{Tr}_{A}\left\{\rho_{C X}^{(\alpha-1) / 2} \rho_{A C X}^{(1-\alpha) / 2} \rho_{A B C X}^{\alpha} \rho_{A C X}^{(1-\alpha) / 2} \rho_{C X}^{(\alpha-1) / 2}\right\}\right)^{1 / \alpha}\right\} .
$$


So

$$
\begin{aligned}
\rho_{C X}^{(\alpha-1) / 2} \rho_{A C X}^{(1-\alpha) / 2} \rho_{A B C X}^{\alpha} \rho_{A C X}^{(1-\alpha) / 2} \rho_{C X}^{(\alpha-1) / 2} & {\left[\sum _ { x } p _ { X } ( x ) \rho _ { C } ^ { x } \otimes | x \rangle \langle x | _ { X } ] ^ { ( \alpha - 1 ) / 2 } \left[\sum_{x} p_{X}(x) \rho_{A C}^{x} \otimes|x\rangle\left\langle\left. x\right|_{X}\right]^{(1-\alpha) / 2} \times\right.\right.} \\
& {\left[\sum _ { x } p _ { X } ( x ) \rho _ { A B C } ^ { x } \otimes | x \rangle \langle x | _ { X } ] ^ { \alpha } \left[\sum_{x} p_{X}(x) \rho_{A C}^{x} \otimes|x\rangle\left\langle\left. x\right|_{X}\right]^{(1-\alpha) / 2} \times\right.\right.} \\
= & {\left[\sum_{x} p_{X}(x) \rho_{C}^{x} \otimes|x\rangle\left\langle\left. x\right|_{X}\right]^{(\alpha-1) / 2}\right.} \\
= & \sum_{x} p_{X}^{\alpha}(x)\left[\rho_{C}^{x}\right]^{(\alpha-1) / 2}\left[\rho_{A C}^{x}\right]^{(1-\alpha) / 2}\left[\rho_{A B C}^{x}\right]^{\alpha}\left[\rho_{A C}^{x}\right]^{(1-\alpha) / 2}\left[\rho_{C}^{x}\right]^{(\alpha-1) / 2} \otimes|x\rangle\left\langle\left. x\right|_{X} .\right.
\end{aligned}
$$

From the fact that

$$
\begin{aligned}
& \operatorname{Tr}\left\{\left(\operatorname{Tr}_{A}\left\{\sum_{x} p_{X}^{\alpha}(x)\left[\rho_{C}^{x}\right]^{(\alpha-1) / 2}\left[\rho_{A C}^{x}\right]^{(1-\alpha) / 2}\left[\rho_{A B C}^{x}\right]^{\alpha}\left[\rho_{A C}^{x}\right]^{(1-\alpha) / 2}\left[\rho_{C}^{x}\right]^{(\alpha-1) / 2} \otimes|x\rangle\left\langle\left. x\right|_{X}\right\}\right)^{1 / \alpha}\right\}\right. \\
& =\operatorname{Tr}\left\{\left(\sum_{x} p_{X}^{\alpha}(x) \operatorname{Tr}_{A}\left\{\left[\rho_{C}^{x}\right]^{(\alpha-1) / 2}\left[\rho_{A C}^{x}\right]^{(1-\alpha) / 2}\left[\rho_{A B C}^{x}\right]^{\alpha}\left[\rho_{A C}^{x}\right]^{(1-\alpha) / 2}\left[\rho_{C}^{x}\right]^{(\alpha-1) / 2}\right\} \otimes|x\rangle\left\langle\left. x\right|_{X}\right)^{1 / \alpha}\right\}\right. \\
& =\operatorname{Tr}\left\{\sum_{x} p_{X}(x)\left(\operatorname{Tr}_{A}\left\{\left[\rho_{C}^{x}\right]^{(\alpha-1) / 2}\left[\rho_{A C}^{x}\right]^{(1-\alpha) / 2}\left[\rho_{A B C}^{x}\right]^{\alpha}\left[\rho_{A C}^{x}\right]^{(1-\alpha) / 2}\left[\rho_{C}^{x}\right]^{(\alpha-1) / 2}\right\}\right)^{1 / \alpha} \otimes|x\rangle\left\langle\left. x\right|_{X}\right\}\right. \\
& =\sum_{x} p_{X}(x) \operatorname{Tr}\left\{\left(\operatorname{Tr}_{A}\left\{\left[\rho_{C}^{x}\right]^{(\alpha-1) / 2}\left[\rho_{A C}^{x}\right]^{(1-\alpha) / 2}\left[\rho_{A B C}^{x}\right]^{\alpha}\left[\rho_{A C}^{x}\right]^{(1-\alpha) / 2}\left[\rho_{C}^{x}\right]^{(\alpha-1) / 2}\right\}\right)^{1 / \alpha}\right\} \\
& =\sum_{x} p_{X}(x) \exp \left\{\left(\frac{\alpha-1}{\alpha}\right) I_{\alpha}(A ; B \mid C)_{\rho^{x}}\right\},
\end{aligned}
$$

it follows that

$$
I_{\alpha}(A ; B \mid C X)_{\rho}=\frac{\alpha}{\alpha-1} \log \sum_{x} p_{X}(x) \exp \left\{\left(\frac{\alpha-1}{\alpha}\right) I_{\alpha}(A ; B \mid C)_{\rho^{x}}\right\} .
$$

\section{B Proof of Conjecture 26 for $\alpha$ in a neighborhood of one}

This section gives more details about proving Conjecture 26 in a neighborhood of one. Consider the inequality in (6.19). Let $\rho, \sigma \in \mathcal{S}(\mathcal{H})_{++}$. We begin by introducing a variable

$$
\gamma=\alpha-1
$$

and with

$$
Y(\gamma) \equiv \rho^{1+\gamma} \sigma^{-\gamma / 2} \mathcal{N}^{\dagger}\left([\mathcal{N}(\sigma)]^{\gamma / 2}[\mathcal{N}(\rho)]^{-\gamma}[\mathcal{N}(\sigma)]^{\gamma / 2}\right) \sigma^{-\gamma / 2}
$$


it follows that $\Delta_{\alpha}(\rho, \sigma, \mathcal{N})$ is equal to

$$
\frac{1}{\alpha-1} \log \operatorname{Tr}\left\{\rho^{\alpha} \sigma^{(1-\alpha) / 2} \mathcal{N}^{\dagger}\left([\mathcal{N}(\sigma)]^{(\alpha-1) / 2}[\mathcal{N}(\rho)]^{1-\alpha}[\mathcal{N}(\sigma)]^{(\alpha-1) / 2}\right) \sigma^{(1-\alpha) / 2}\right\}=\frac{1}{\gamma} \log \operatorname{Tr}\{Y(\gamma)\} .
$$

Since $d \gamma / d \alpha=1$,

$$
\begin{array}{r}
\frac{d}{d \alpha}\left[\frac{1}{\alpha-1} \log \operatorname{Tr}\left\{\rho^{\alpha} \sigma^{(1-\alpha) / 2} \mathcal{N}^{\dagger}\left([\mathcal{N}(\sigma)]^{(\alpha-1) / 2}[\mathcal{N}(\rho)]^{1-\alpha}[\mathcal{N}(\sigma)]^{(\alpha-1) / 2}\right) \sigma^{(1-\alpha) / 2}\right\}\right] \\
=\frac{d}{d \gamma}\left[\frac{1}{\gamma} \log \operatorname{Tr}\{Y(\gamma)\}\right] .
\end{array}
$$

We can then explicitly compute the derivative:

$$
\begin{aligned}
\frac{d}{d \gamma}\left[\frac{1}{\gamma} \log \operatorname{Tr}\{Y(\gamma)\}\right] & =-\frac{1}{\gamma^{2}} \log \operatorname{Tr}\{Y(\gamma)\}+\frac{\operatorname{Tr}\left\{\frac{d}{d \gamma} Y(\gamma)\right\}}{\gamma \operatorname{Tr}\{Y(\gamma)\}} \\
& =\frac{\gamma \operatorname{Tr}\left\{\frac{d}{d \gamma} Y(\gamma)\right\}-\operatorname{Tr}\{Y(\gamma)\} \log \operatorname{Tr}\{Y(\gamma)\}}{\gamma^{2} \operatorname{Tr}\{Y(\gamma)\}} .
\end{aligned}
$$

We can prove that the numerator of $(\overline{B .6})$ is non-negative for $\gamma$ in a neighborhood of zero. To this end, consider a Taylor expansion of $Y(\gamma)$ in (B.2) around $\gamma$ equal to zero (so around $\alpha$ equal to one). Indeed, consider that

$$
\begin{aligned}
X^{1+\gamma} & =X+\gamma X \log X+\frac{\gamma^{2}}{2} X \log ^{2} X+O\left(\gamma^{3}\right), \\
X^{\gamma} & =I+\gamma \log X+\frac{\gamma^{2}}{2} \log ^{2} X+O\left(\gamma^{3}\right) .
\end{aligned}
$$

For our case, we make the following substitutions into $\operatorname{Tr}\{Y(\gamma)\}$ :

$$
\begin{aligned}
\rho^{1+\gamma} & =\rho+\gamma \rho \log \rho+\frac{\gamma^{2}}{2} \rho \log ^{2} \rho+O\left(\gamma^{3}\right), \\
\sigma^{-\gamma / 2} & =I-\frac{\gamma}{2} \log \sigma+\frac{\gamma^{2}}{8} \log ^{2} \sigma+O\left(\gamma^{3}\right), \\
{[\mathcal{N}(\sigma)]^{\gamma / 2} } & =I+\frac{\gamma}{2} \log [\mathcal{N}(\sigma)]+\frac{\gamma^{2}}{8} \log ^{2}[\mathcal{N}(\sigma)]+O\left(\gamma^{3}\right), \\
{[\mathcal{N}(\rho)]^{-\gamma} } & =I-\gamma \log [\mathcal{N}(\rho)]+\frac{\gamma^{2}}{2} \log ^{2}[\mathcal{N}(\rho)]+O\left(\gamma^{3}\right) .
\end{aligned}
$$

After a rather tedious calculation, we find that

$$
\operatorname{Tr}\{Y(\gamma)\}=\operatorname{Tr}\{\rho\}+\gamma \Delta(\rho, \sigma, \mathcal{N})+\frac{\gamma^{2}}{2}\left[V(\rho, \sigma, \mathcal{N})+[\Delta(\rho, \sigma, \mathcal{N})]^{2}\right]+O\left(\gamma^{3}\right),
$$

where $\Delta(\rho, \sigma, \mathcal{N})$ is the relative entropy difference (of the form written in (6.4) ) and $V(\rho, \sigma, \mathcal{N})$ is a relative entropy difference variance of $\rho, \sigma$, and $\mathcal{N}$ :

$$
\begin{aligned}
& V(\rho, \sigma, \mathcal{N}) \equiv \operatorname{Tr}\left\{\rho\left[\log \rho-\log \sigma-\mathcal{N}^{\dagger}(\log \mathcal{N}(\rho))+\mathcal{N}^{\dagger}(\log \mathcal{N}(\sigma))-\Delta(\rho, \sigma, \mathcal{N})\right]^{2}\right\} \\
& \quad+\operatorname{Tr}\left\{\mathcal{N}(\rho)[\log \mathcal{N}(\rho)-\log \mathcal{N}(\sigma)]^{2}\right\}-\operatorname{Tr}\left\{\rho\left[\mathcal{N}^{\dagger}(\log \mathcal{N}(\rho))-\mathcal{N}^{\dagger}(\log \mathcal{N}(\sigma))\right]^{2}\right\}
\end{aligned}
$$


Letting $A=\log \mathcal{N}(\rho)-\log \mathcal{N}(\sigma)$, observe that

$$
\begin{aligned}
& \operatorname{Tr}\left\{\mathcal{N}(\rho)[\log \mathcal{N}(\rho)-\log \mathcal{N}(\sigma)]^{2}\right\}-\operatorname{Tr}\left\{\rho\left[\mathcal{N}^{\dagger}(\log \mathcal{N}(\rho))-\mathcal{N}^{\dagger}(\log \mathcal{N}(\sigma))\right]^{2}\right\} \\
& =\operatorname{Tr}\left\{\mathcal{N}(\rho) A^{\dagger} A\right\}-\operatorname{Tr}\left\{\rho \mathcal{N}^{\dagger}\left(A^{\dagger}\right) \mathcal{N}^{\dagger}(A)\right\} \\
& =\operatorname{Tr}\left\{\rho \mathcal{N}^{\dagger}\left(A^{\dagger} A\right)\right\}-\operatorname{Tr}\left\{\rho \mathcal{N}^{\dagger}\left(A^{\dagger}\right) \mathcal{N}^{\dagger}(A)\right\} \\
& =\operatorname{Tr}\left\{\rho\left[\mathcal{N}^{\dagger}\left(A^{\dagger} A\right)-\mathcal{N}^{\dagger}\left(A^{\dagger}\right) \mathcal{N}^{\dagger}(A)\right]\right\} \\
& \geq 0 .
\end{aligned}
$$

The inequality follows by applying the Kadison-Schwarz inequality [Bha07, i.e., for any 2-positive unital map $\phi$, we have that

$$
\phi\left(a^{\dagger} a\right) \geq \phi\left(a^{\dagger}\right) \phi(a) .
$$

For any Hermitian operator $H$, we have that

$$
\left\langle H^{2}\right\rangle_{\rho}-\langle H\rangle_{\rho}^{2} \geq 0
$$

So taking $H \equiv \log \rho-\log \sigma-\mathcal{N}^{\dagger} \log \mathcal{N}(\rho)+\mathcal{N}^{\dagger} \log \mathcal{N}(\sigma)$, we conclude that

$$
\operatorname{Tr}\left\{\rho\left[\log \rho-\log \sigma-\mathcal{N}^{\dagger}(\log \mathcal{N}(\rho))+\mathcal{N}^{\dagger}(\log \mathcal{N}(\sigma))-\Delta(\rho, \sigma, \mathcal{N})\right]^{2}\right\} \geq 0 .
$$

Putting the two inequalities above together, we conclude that $V(\rho, \sigma, \mathcal{N}) \geq 0$, an observation central to our development here.

We will make the abbreviations $\Delta \equiv \Delta(\rho, \sigma, \mathcal{N})$ and $V \equiv V(\rho, \sigma, \mathcal{N})$ from here forward, so that

$$
\operatorname{Tr}\{Y(\gamma)\}=1+\gamma \Delta+\frac{\gamma^{2}}{2}\left[V+\Delta^{2}\right]+O\left(\gamma^{3}\right) .
$$

So this implies that

$$
\gamma \operatorname{Tr}\left\{\frac{d}{d \gamma} Y(\gamma)\right\}=\gamma \Delta+\gamma^{2}\left[V+\Delta^{2}\right]+O\left(\gamma^{3}\right)
$$

$\operatorname{Tr}\{Y(\gamma)\} \log \operatorname{Tr}\{Y(\gamma)\}=\left[1+\gamma \Delta+\frac{\gamma^{2}}{2}\left[V+\Delta^{2}\right]+O\left(\gamma^{3}\right)\right] \log \left[1+\gamma \Delta+\frac{\gamma^{2}}{2}\left[V+\Delta^{2}\right]+O\left(\gamma^{3}\right)\right]$.

Then for small $\gamma$, we have the following Taylor expansion for the logarithm:

$$
\begin{aligned}
\log \left[1+\gamma \Delta+\frac{\gamma^{2}}{2}\left[V+\Delta^{2}\right]+O\left(\gamma^{3}\right)\right] & =\gamma \Delta+\frac{\gamma^{2}}{2}\left[V+\Delta^{2}\right]-\frac{\gamma^{2} \Delta^{2}}{2}+O\left(\gamma^{3}\right) \\
& =\gamma \Delta+\frac{\gamma^{2}}{2} V+O\left(\gamma^{3}\right)
\end{aligned}
$$

which gives

$$
\begin{aligned}
\operatorname{Tr}\{Y(\gamma)\} \log \operatorname{Tr}\{Y(\gamma)\} & =\left[1+\gamma \Delta+\frac{\gamma^{2}}{2}\left[V+\Delta^{2}\right]+O\left(\gamma^{3}\right)\right]\left[\gamma \Delta+\frac{\gamma^{2}}{2} V+O\left(\gamma^{3}\right)\right] \\
& =\gamma \Delta+\frac{\gamma^{2}}{2} V+\gamma^{2} \Delta^{2}+O\left(\gamma^{3}\right)
\end{aligned}
$$


Finally, we can say that

$$
\begin{aligned}
\gamma \operatorname{Tr}\left\{\frac{d}{d \gamma} Y(\gamma)\right\}-\operatorname{Tr}\{Y(\gamma)\} \log \operatorname{Tr}\{Y(\gamma)\} & =\gamma \Delta+\gamma^{2}\left[V+\Delta^{2}\right]-\left[\gamma \Delta+\frac{\gamma^{2}}{2} V+\gamma^{2} \Delta^{2}\right]+O\left(\gamma^{3}\right) \\
& =\frac{\gamma^{2}}{2} V+O\left(\gamma^{3}\right) .
\end{aligned}
$$

If $V>0$, we can conclude that as long as $\gamma$ is very near to zero, all terms $O\left(\gamma^{3}\right)$ are negligible in comparison to $\frac{\gamma^{2}}{2} V$, and the monotonicity holds in such a regime. (Note that this argument does not work if $V=0$.)

A similar kind of development can also be used to show that the the inequality in (6.20) holds for $\gamma$ in a neighborhood of zero (i.e., $\alpha$ in a neighborhood of one).

\section{References}

[AF04] Robert Alicki and Mark Fannes. Continuity of quantum conditional information. Journal of Physics A: Mathematical and General, 37(5):L55, February 2004. arXiv:quantph/0312081.

[AH13] Koenraad M.R. Audenaert and Fumio Hiai. On matrix inequalities between the power means: Counterexamples. Linear Algebra and its Applications, 439(5):1590-1604, September 2013. arXiv:1302.7040.

[BBPS96] Charles H. Bennett, Herbert J. Bernstein, Sandu Popescu, and Benjamin Schumacher. Concentrating partial entanglement by local operations. Physical Review A, 53(4):2046-2052, April 1996. arXiv:quant-ph/9511030.

[BCY11] Fernando G.S.L. Brandao, Matthias Christandl, and Jon Yard. Faithful squashed entanglement. Communications in Mathematical Physics, 306(3):805-830, September 2011. arXiv:1010.1750.

[BDSW96] Charles H. Bennett, David P. DiVincenzo, John A. Smolin, and William K. Wootters. Mixed-state entanglement and quantum error correction. Physical Review A, 54(5):3824-3851, November 1996. arXiv:quant-ph/9604024.

[Bha07] Rajendra Bhatia. Positive Definite Matrices. Princeton University Press, 2007.

[BSW15a] Mario Berta, Kaushik Seshadreesan, and Mark M. Wilde. Rényi generalizations of the conditional quantum mutual information. Journal of Mathematical Physics, 56(2):022205, February 2015. arXiv:1403.6102.

[BSW15b] Mario Berta, Kaushik P. Seshadreesan, and Mark M. Wilde. Rényi generalizations of quantum information measures. Physical Review A, 91(2):022333, February 2015. arXiv:1502.07977.

[CL14] Eric A. Carlen and Elliott H. Lieb. Remainder terms for some quantum entropy inequalities. Journal of Mathematical Physics, 55(4):042201, April 2014. arXiv:1402.3840. 
[CMW14] Thomas Cooney, Milan Mosonyi, and Mark M. Wilde. Strong converse exponents for a quantum channel discrimination problem and quantum-feedback-assisted communication. August 2014. arXiv:1408.3373.

[Cub09] Toby Cubitt. Matlab quantum information package (quantinf v0.5.1). http://www.dr-qubit.org/matlab.php, 2009.

[CW04] Matthias Christandl and Andreas Winter. "Squashed entanglement": An additive entanglement measure. Journal of Mathematical Physics, 45(3):829-840, March 2004. arXiv:quant-ph/0308088.

[Dat09] Nilanjana Datta. Min- and max-relative entropies and a new entanglement monotone. IEEE Transactions on Information Theory, 55(6):2816-2826, June 2009. arXiv:0803.2770.

[DY08] Igor Devetak and Jon Yard. Exact cost of redistributing multipartite quantum states. Physical Review Letters, 100(23):230501, June 2008.

[FNW15] Motohisa Fukuda, Ion Nechita, and Michael M. Wolf. Quantum channels with polytopic images and image additivity. IEEE Transactions on Information Theory, 61(4):1851-1859, April 2015. arXiv:1408.2340.

[FR14] Omar Fawzi and Renato Renner. Quantum conditional mutual information and approximate Markov chains. October 2014. arXiv:1410.0664.

[GPW05] Berry Groisman, Sandu Popescu, and Andreas Winter. Quantum, classical, and total amount of correlations in a quantum state. Physical Review A, 72(3):032317, September 2005. arXiv:quant-ph/0410091.

[GW15] Manish K. Gupta and Mark M. Wilde. Multiplicativity of completely bounded $p$ norms implies a strong converse for entanglement-assisted capacity. Communications in Mathematical Physics, 334(2):867-887, March 2015. arXiv:1310.7028.

[HHHH09] Ryszard Horodecki, Paweł Horodecki, Michał Horodecki, and Karol Horodecki. Quantum entanglement. Reviews of Modern Physics, 81(2):865-942, June 2009. arXiv:quant-ph/0702225v2.

[HJPW04] Patrick Hayden, Richard Jozsa, Denes Petz, and Andreas Winter. Structure of states which satisfy strong subadditivity of quantum entropy with equality. Communications in Mathematical Physics, 246(2):359-374, April 2004. arXiv:quant-ph/0304007.

[Hol73] Alexander S. Holevo. Bounds for the quantity of information transmitted by a quantum communication channel. Probl. Peredachi Inf., 9(3):3-11, 1973.

[HOW05] Michal Horodecki, Jonathan Oppenheim, and Andreas Winter. Partial quantum information. Nature, 436:673-676, August 2005.

[HOW07] Michal Horodecki, Jonathan Oppenheim, and Andreas Winter. Quantum state merging and negative information. Communications in Mathematical Physics, 269(1):107136, January 2007. arXiv:quant-ph/0512247. 
[Kim13] Isaac H. Kim. Application of conditional independence to gapped quantum many-body systems. http://www.physics.usyd.edu.au/quantum/Coogee2013, January 2013. Slide 43.

[KW04] Masato Koashi and Andreas Winter. Monogamy of quantum entanglement and other correlations. Physical Review A, 69(2):022309, February 2004. arXiv:quant$\mathrm{ph} / 0310037$.

[Lin74] Göran Lindblad. Expectations and entropy inequalities for finite quantum systems. Communications in Mathematical Physics, 39(2):111-119, July 1974.

[Lin75] Göran Lindblad. Completely positive maps and entropy inequalities. Communications in Mathematical Physics, 40(2):147-151, June 1975.

$\left[\mathrm{MBC}^{+} 12\right]$ Kavan Modi, Aharon Brodutch, Hugo Cable, Tomasz Paterek, and Vlatko Vedral. The classical-quantum boundary for correlations: Discord and related measures. Reviews of Modern Physics, 84(4):1655-1707, November 2012. arXiv:1112.6238.

$\left[\mathrm{MBP}^{+} 15\right]$ Avijit Misra, Anindya Biswas, Arun K. Pati, Aditi Sen(De), and Ujjwal Sen. Quantum correlation with sandwiched relative entropies: Advantageous as order parameter in quantum phase transitions. Phys. Rev. E, 91:052125, May 2015. arXiv:1406.5065v1.

[MH11] Milán Mosonyi and Fumio Hiai. On the quantum Rényi relative entropies and related capacity formulas. IEEE Transactions on Information Theory, 57(4):2474-2487, April 2011. arXiv:0912.1286.

[MLDS $\left.{ }^{+} 13\right]$ Martin Müller-Lennert, Frédéric Dupuis, Oleg Szehr, Serge Fehr, and Marco Tomamichel. On quantum Rényi entropies: a new definition and some properties. Journal of Mathematical Physics, 54(12):122203, December 2013. arXiv:1306.3142.

[MO15] Milan Mosonyi and Tomohiro Ogawa. Quantum hypothesis testing and the operational interpretation of the quantum rényi relative entropies. Communications in Mathematical Physics, 334(3):1617-1648, 2015. arXiv:1309.3228.

[OZ01] Harold Ollivier and Wojciech H. Zurek. Quantum discord: A measure of the quantumness of correlations. Physical Review Letters, 88(1):017901, December 2001. arXiv:quant-ph/0105072.

[Pet86] Dénes Petz. Quasi-entropies for finite quantum systems. Reports in Mathematical Physics, 23(1):57-65, February 1986.

[Pia12] Marco Piani. Problem with geometric discord. Physical Review A, 86(3):034101, September 2012. arXiv:1206.0231.

[PV07] Martin B. Plenio and Shashank Virmani. An introduction to entanglement measures. Quantum and Information Computation, 7(1):1-51, January 2007. arXiv:quant$\mathrm{ph} / 0504163 \mathrm{v} 3$.

[Ren05] Renato Renner. Security of Quantum Key Distribution. PhD thesis, ETH Zürich, December 2005. arXiv:quant-ph/0512258. 
[Sch95] Benjamin Schumacher. Quantum coding. Physical Review A, 51(4):2738-2747, April 1995.

[Suz85] Masuo Suzuki. Transfer-matrix method and Monte Carlo simulation in quantum spin systems. Physical Review B, 31(5):2957, March 1985.

[SW13] Naresh Sharma and Naqueeb Ahmad Warsi. Fundamental bound on the reliability of quantum information transmission. Phys. Rev. Lett., 110:080501, Feb 2013. arXiv:1205.1712.

[SW14] Kaushik P. Seshadreesan and Mark M. Wilde. Fidelity of recovery, geometric squashed entanglement, and measurement recoverability. October 2014. arXiv:1410.1441.

[TCR09] Marco Tomamichel, Roger Colbeck, and Renato Renner. A fully quantum asymptotic equipartition property. IEEE Transactions on Information Theory, 55(12):5840-5847, December 2009. arXiv:0811.1221.

[TGW14a] Masahiro Takeoka, Saikat Guha, and Mark M. Wilde. Fundamental rate-loss tradeoff for optical quantum key distribution. Nature Communications, 5:5235, October 2014. arXiv:1504.06390.

[TGW14b] Masahiro Takeoka, Saikat Guha, and Mark M. Wilde. The squashed entanglement of a quantum channel. IEEE Transactions on Information Theory, 60(8):4987-4998, August 2014. arXiv:1310.0129.

[Tom12] Marco Tomamichel. A Framework for Non-Asymptotic Quantum Information Theory. PhD thesis, ETH Zurich, 2012. arXiv:1203.2142.

[Tou14] Dave Touchette. Quantum information complexity and amortized communication. April 2014. arXiv:1404.3733.

[TWW14] Marco Tomamichel, Mark M. Wilde, and Andreas Winter. Strong converse rates for quantum communication. June 2014. arXiv:1406.2946.

[Vid00] Guifre Vidal. Entanglement monotones. Journal of Modern Optics, 47:355, 2000. arXiv:quant-ph/9807077.

[WL12] Andreas Winter and $\mathrm{Ke}$ Li. A stronger subadditivity relation? http://www.maths.bris.ac.uk/ csajw/stronger_subadditivity.pdf, 2012.

[WWY14] Mark M. Wilde, Andreas Winter, and Dong Yang. Strong converse for the classical capacity of entanglement-breaking and Hadamard channels via a sandwiched Rényi relative entropy. Communications in Mathematical Physics, 331(2):593-622, October 2014. arXiv:1306.1586.

[YD09] Jon Yard and Igor Devetak. Optimal quantum source coding with quantum side information at the encoder and decoder. IEEE Transactions in Information Theory, 55(11):5339-5351, November 2009. arXiv:0706.2907.

[Zha14] Lin Zhang. A stronger monotonicity inequality of quantum relative entropy: A unifying approach via Rényi relative entropy. March 2014. arXiv:1403.5343. 
[Zur00] Wojciech H. Zurek. Einselection and decoherence from an information theory perspective. Annalen der Physik, 9(11-12):855-864, November 2000. arXiv:quant-ph/0011039.

[ZW14] Lin Zhang and Junde Wu. A lower bound of quantum conditional mutual information. Journal of Physics A: Mathematical and Theoretical, 47(41):415303, October 2014. arXiv:1403.1424. 